\title{
Fostering breakthrough technologies - How do optimal funding decisions depend on evaluation accuracy?
}

\author{
Eeva Vilkkumaa ${ }^{\mathrm{a}, *}$, Ahti Salo ${ }^{\mathrm{a}}$, Juuso Liesiö ${ }^{\mathrm{b}}$, Afzal Siddiqui ${ }^{\mathrm{c}, \mathrm{d}}$ \\ ${ }^{a}$ Department of Mathematics and Systems Analysis, Aalto University School of Science, \\ P.O. Box 11100, 00076 Aalto, Finland \\ ${ }^{b}$ Department of Information and Service Economy, Aalto University School of Business, \\ P.O. Box 21220, 00076 Aalto, Finland \\ ${ }^{c}$ Department of Statistical Science, University College London, \\ Gower Street, London WC1E 6BT, United Kingdom \\ ${ }^{d}$ Department of Computer and Systems Sciences, Stockholm University, \\ DSV, Borgarfjordsgatan 15, SE-164 40 Kista, Sweden
}

\begin{abstract}
There is growing interest in fostering breakthrough technologies that offer exceptionally high value to society. However, when starting technology projects, it is impossible to know which of them have the potential to lead to breakthroughs. Therefore, organizations have adopted funding policies in which ongoing projects are subjected to interim evaluations based on which some projects may be abandoned to release resources for seizing new opportunities. In this paper, we study which funding policies are optimal when the objective is either (i) to maximize the expected value of the project portfolio, or (ii) to maximize the expected number of exceptionally excellent projects that may lead to breakthrough technologies. We show that the optimal policy for funding exceptionally excellent projects is to start a large number of projects and abandon a high proportion of them later, whereas the optimal policy for maximizing the expected value of the project portfolio is to grant long-term funding to a smaller set of projects based on initial evaluation. Furthermore, we show how the trade-off between these two objectives depends on the initial project evaluation accuracy
\end{abstract}

\footnotetext{
* Corresponding author

Email addresses: eeva.vilkkumaa@aalto.fi (Eeva Vilkkumaa), ahti.salo@aalto.fi (Ahti Salo), juuso.liesio@aalto.fi (Juuso Liesiö), afzal.siddiqui@ucl.ac.uk (Afzal Siddiqui)
} 
and the rate at which this accuracy improves. Our results suggest that this trade-off is particularly significant when the initial project evaluations are very uncertain but become more accurate soon after the projects have been launched. In such a setting, policies that seek to maximize the expected portfolio value may fail to promote breakthrough technologies.

Keywords: breakthrough technologies, project portfolio selection, abandonment option

\section{Introduction}

$\mathcal{T}$ Fostering of breakthrough technologies has in recent years become one of the key objectives of many organizations, such as governmental institutions and public research funding agencies. The policy interest in breakthrough tech-

5 nologies stems from the potential of such technologies for creating extensive industrial development, enhancing national competitiveness, and generating employment and export growth (Sharpe et al., 2013). Moreover, breakthrough technologies may result in the establishment of 'new technology platforms' with applications across a range of products and markets. For instance, Liquid Crystal Displays (LCDs) developed in the 1960s have since grown into a global industry with applications ranging from pocket calculators to televisions and laptops. Also, fiber-optic communication systems developed in the 1970s, together with successive waves of innovation in optical fibers and fiber amplifiers in the 1980s, have accelerated the expansion of the Internet age by allowing 15 huge amounts of data to be transmitted.

Breakthrough technologies such as those mentioned above are extremely rare. Therefore, the objective of promoting breakthrough technologies is typically pursued by trying to identify and fund exceptionally excellent technologyrelated activities which have the potential to result in breakthroughs. Governments, for instance, invest in training highly skilled staff for research laboratories, provide R\&D subsidies and grants to private companies and public research institutes, and act as first customers for new technologies through public 
procurement (Sharpe et al., 2013). Many research funding agencies have dedicated programs for supporting exceptional excellence; the National Institutes of

25 Health (NIH), for instance, have established a high-risk, high-reward research program for 'scientists of exceptional creativity who propose highly innovative approaches to major contemporary challenges in biomedical research' (NIH, 2014). The European Research Council (ERC), too, seeks 'to support the best of the best scientific effort in Europe', expecting that 'its grants will help to bring about new and unpredictable scientific discoveries - the kind that can form the basis of new industries, markets, and broader social innovations of the future' (ERC, 2014).

Decisions about which technology-related activities (henceforth referred to as projects) to fund are typically based on evaluation of project proposals. At the

35 time of launching a project, however, it is usually impossible to know whether the project will be exceptionally excellent. Thus, to avoid the prospect of committing resources to projects that will ultimately fail, organizations have adopted flexible funding policies in which on-going projects are subjected to interim evaluations and, based on these evaluations, some projects are abandoned

40 to release resources for seizing fresh opportunities (O'Connor et al., 2008; Tellis et al., 2009; Tian, 2011). The value of such flexibility, called the abandonment option in the real options literature (Dixit and Pindyck, 1994), has been studied extensively in contexts where the objective is to maximize the expected value of the project or project portfolio (e.g., Roberts and Weitzman, 1981; Huchzer45 meier and Loch, 2001; Gustafsson and Salo, 2005; Santiago and Vakili, 2005). Yet, to our knowledge no quantitative models have been developed to support the shaping of policies that promote breakthrough technologies through funding exceptionally excellent projects.

In this paper, we develop a multi-period project portfolio selection model so and, specifically, establish guidelines for the optimal funding, evaluation, and abandonment of projects when the objective is either (i) to maximize the expected value offered by the projects or (ii) to maximize the expected number of projects which have exceptionally high values ex post exceeding a given threshold 
level. We establish these guidelines by solving a two-stage stochastic program-

${ }_{55}$ ming problem in which the discrete decision variables consist of (i) the number of projects that are launched, re-evaluated, and abandoned and (ii) the number of periods for which projects will be funded before they are re-evaluated. We also derive analytic necessary conditions for optimal funding policies, which allows us to solve the optimization problems with a reasonable computational 60 effort.

The results of our model suggest that to maximize the expected value of the project portfolio, one should provide full funding to those technology projects which appear the best based on the initial evaluation. This policy differs from the optimal policy for promoting breakthrough technologies through funding

${ }_{65}$ exceptionally excellent projects, which is to launch a large number of projects, re-evaluate most of them after some time and, based on the resulting information, abandon a high proportion of on-going projects. The trade-off between these two objectives is shown to depend largely on the accuracy of the initial project evaluations and the rate at which this accuracy is improved. This trade-

7o off is particularly significant, if the initial project evaluations are very uncertain but become more accurate quickly after the projects have been launched. The important implication of these results is that policies which seek to maximize the expected portfolio value may fall far short of promoting breakthrough technologies, and vice versa.

75 2. Related Literature

Arthur (2009) defines a technology as 'a collection of phenomena captured and put to use'. Breakthrough technologies are defined by Sharpe et al. (2013) as 'novel and discontinuous innovations that result in significant and irreversible changes and are based on new, under- or unexploited physical, chemical, and so biological phenomena that allow order of magnitude improvements in the performance of existing products and/or the creation of entirely new ones'. The potential for large improvements compared to existing practices is also captured 
by related terms that are often used interchangeably in high technology management literature, such as radical innovation (Utterback, 1994) and disruptive s5 technology (Christensen, 1997).

The promotion of breakthrough technologies and radical innovation has so far been examined mostly through empirical studies. O'Connor et al. (2008), for instance, use data from 85 individuals involved with innovation efforts in large firms and conclude that real options approaches and experimental learnstrong positive effects on innovation success. Klingebiel and Rammer (2011) study the innovation performance of 1500 German companies and note better performance among those firms that allocate resources to a broad range of projects and terminate those with unfavorable prospects. Tellis et al. (2009), building on survey and archival data of 750 firms worldwide, conclude that the 95 strongest drivers for radical innovation include the willingness to cannibalize on the firm's current assets to get ahead with the next generation of innovation; the ability to realize the limitations of the current technology and the emergence of a dominant one; and the tolerance for risks associated with trading the current, certain profit stream for a new, uncertain one.

From the perspective of public policy, there is plenty of research on the right 'mix' of policy instruments to support the performance of the innovation system (Borrás and Equist, 2013; Marxt and Brunner, 2013; Sharpe et al., 2013). Due to institutional factors, though, the effectiveness of such instruments - including government incubators, seed funding, and loan guarantees - may vary greatly between different countries (Hall and Lerner, 2009). As a general guideline, Lerner (2009) suggests that the policy instruments should be sufficiently preserving but, on the other hand, their efficacy should be regularly monitored so that inefficient policy instruments could be abandoned or modified to meet the needs of the changing market environment. In the context of research funding, similar conclusions have been made regarding the need for a balance between committing to research projects for a sufficiently long period of time on the one hand, and being able to seize emerging opportunities on the other hand. In particular, short-term funding has been found to encourage risk averse research 
strategies and to generate proximate and often predictable outcomes, while high impact research seems to be connected to the explorative mode conducted using long-term funding (Bourke and Butler, 1999; Heinze, 2008). Due to scarce resources, however, the provision of long-term funding necessitates highly selective funding processes which, in turn, increase the risk of failing to fund projects that could have resulted in breakthroughs (Melin and Danell, 2006).

Although empirical studies such as these have been carried out, no quantitative models of project selection have been presented to guide the shaping of optimal policies for promoting breakthrough technologies through funding exceptionally excellent projects. Instead, the objective of most quantitative project selection methodologies is to maximize the expected value or utility. The traditional approach is to compute the (expected) net present value (NPV) for each project and to start those projects that have a positive NPV. Since the 1990s, however, this 'now-or-never' type NPV approach has been increasingly complemented and partly replaced by the theory of real options, which helps determine how much additional value can be gained by exploiting different kinds of managerial flexibility. This flexibility can result, for instance, from the option to postpone the investment until market conditions become more favorable, or to abandon an on-going project if its interim results fall short of expectations (see Dixit and Pindyck 1994 for an overview).

The classic result in real options theory is that the more uncertain the projects' future benefits are, the higher the value of the real option and the greater the delay in taking action (Roberts and Weitzman, 1981; Dixit and Pindyck, 1994; Alvarez, 2001). Relationships between different kinds of options and sources of uncertainty have been studied extensively in decision contexts such as oil and gas investments (Smith and McCardle, 1999), new product development (Huchzermeier and Loch, 2001; Santiago and Vakili, 2005), and development of renewable energy technologies (Siddiqui et al., 2007).

Real options analysis helps decide whether and when to invest. In doing so, most real options approaches assume that (i) there is no strict budget constraint so that all good projects can be funded and (ii) the value of each project can 
be observed at any given time. These assumptions are fairly realistic when considering $\mathrm{R} \& \mathrm{D}$ investment decisions in large private companies, for instance. In this paper, however, we examine a decision setting that is typically faced by public organizations, in which (i) there is a fixed budget for each funding period that is to be allocated among projects with known costs but uncertain benefits and (ii) the decision maker has limited possibilities for monitoring the progress of on-going projects. In this setting, the question is rather in which projects to invest - in other words, which project portfolio to select - and when to revisit the investment decision to release resources for new project opportunities. Such project portfolio selection problems can be supported by methods of portfolio decision analysis (see Salo et al. 2011 for an overview), which is the modeling approach adopted in this paper.

\section{Model Framework}

\subsection{Model description}

We consider a multi-period decision process in which the decision-maker, at the beginning of each period, allocates a fixed budget to a portfolio of technology projects. This portfolio is selected based on estimates about the projects' future values. This 'future value' refers to the extent to which the project generates results that contribute to the attainment of the decision-maker's objectives. For instance, the future value could be interpreted as the number of patents resulting from the project or, more broadly, as the increase in technological capabilities relative to the existing technological frontier.

The future value will be realized at the time when the project is completed, i.e., at the end of its planned duration. If a project is abandoned prior to completion, then some value may nevertheless be generated. This salvage value 170 (Pasternack, 1985; Alvarez, 2001) can represent improved expertise, new collaborative networks, or an early product prototype built by the time of abandonment (Roberts and Weitzman, 1981). 
An exceptionally excellent project (henceforth referred to as exceptional project) is here defined as one which has an exceptionally high future value. 175 More specifically, a project is interpreted as an exceptional project if its future value is in the upper tail of the prior distribution of the population of project values such that the probability mass of this tail corresponds to the desired level of excellence $\alpha$, e.g., the top $1 \%$ or the top $5 \%$. This level of excellence, together with the prior distribution for the future values, determines the excellence threshold for the value that the project must deliver in order to qualify as an exceptional project (see Figure 1 for illustration).

Insert Figure 1 about here.

Defining exceptional projects through a threshold on the projets' future values captures the intuition that for an emerging technology to succeed, its limit must surpass those of traditional technologies (Brown, 1992; Adner and Levinthal, 2002). This definition parallels that of Öquist and Benner (2012), who define breakthrough research as the most highly-cited $10 \%$ among scientific papers worldwide. Huchzermeier and Loch (2001) use an analogous threshold to model the minimum performance required by the market for a project to yield a premium profit margin.

Exceptional projects are assumed to have the potential to result in the development of breakthrough technologies. The policy interest in breakthrough technologies stems from the recognition that they yield extremely high, indirect societal benefits, which are sometimes realized only long after the projects have been completed. For instance, the invention of the microprocessor in the early 1970s led to transformative societal changes by facilitating the development of low-cost personal computers and mobile phones. Also, the invention of the scanning tunnelling microscope in 1981 and its further development through the invention of the atomic force microscope in 1986 were fundamental to the subsequent development of nanotechnology, which is widely seen as the driving force behind a new industrial revolution (OECD, 2010). 
We assume that the indirect benefits potentially resulting from an exceptional technology project will not be realized unless the project is completed. This condition reflects the empirical finding that breakthrough innovations tend to require long-term funding to succeed (Heinze, 2008; Azoulay et al., 2011). The indirect benefits may, thus, be enabled only if (i) the project is completed and (ii) its future value is in the top $\alpha$-tail of the prior distribution for these values. Nevertheless, the indirect benefits are not captured by the prior distribution (such as that illustrated in Figure 1) but, instead are much higher than any value that is likely to result from this distribution.

At the time of launching projects, estimates about their future value are uncertain. We assume that the decision-maker has the option to re-evaluate on-going projects after some time at a given cost and, based on more recent information, to abandon some of these projects, which in turn frees resources so that more new projects can be launched. On this basis, we develop a project selection and evaluation model to study the multi-period project portfolio selection process with such re-evaluation and abandonment options. This is done from the perspective of two different objectives: (i) the maximization of the expected future value of the selected portfolio, and (ii) the maximization of the expected number of exceptional projects in the portfolio. For each of these objectives, we address the following research questions:

- How should the total budget be divided between project funding and evaluation costs?

- When should interim evaluations about the projects be acquired?

- What is the value of the option to re-evaluate and abandon projects?

By addressing these questions, we provide guidelines to support the development of optimal policies for project evaluation and funding when the objective is to maximize either the expected future portfolio value or the number of exceptional projects in the portfolio. For this purpose, instead of focusing on a single realization of a portfolio selection problem, we examine how the multi-period 
decision process can be expected to unfold over time. Accordingly, we do not intend to optimize the funding policy for each period separately but, instead, determine a static funding policy that would, on average, yield the highest portfolio value or the highest number of exceptional projects over time. While the use of a static funding policy may seem rigid from the point of view of private companies, the funding policies of public organizations are often fixed within a relatively static frame for longer periods of time due to various administrative reasons.

We assume that the technology projects are independent in the sense that the future value of a given project does not depend on what other projects are underway. Practically all technologies complement existing technologies by, for instance, building on them (Arthur, 2009) or utilizing them as complementary assets required for generating profits (Teece, 1986); such complementarities are, however, assumed to be captured by the projects' future values. The case in which these future values are independent of one another serves as a useful benchmark and makes it possible to obtain some analytic results on optimal funding policies. Nevertheless, interdependencies between projects' values could be modeled by introducing 'dummy' projects whose values would only be realized if each of the interdependent projects was funded (Liesiö et al., 2008).

To facilitate the interpretation of our results by keeping the model sufficiently parsimonious, we also make the following simplifying assumptions, each of which could be relaxed by relatively simple modifications: (i) there are equally many project proposals in each period, (ii) the projects are of equal duration, (iii) each project, if funded, consumes one resource unit per period, and (iv) 255 the probability distributions that characterize the projects' future values and estimates about these values do not change over time.

\subsection{Model formulation}

\subsubsection{Feasible funding policies}

We assume that $n$ new project proposals arrive at the beginning of funding period $t$. The duration of each project is $d$ periods, meaning that a project 
launched at the beginning of period $t$ is completed at the end of period $t+$ $d-1$. Depending on the time span of the industry, the length of one period can be different, varying from half a year to several years, for instance. Out of $n$ proposals, the decision-maker launches $\ell$ projects of which $e$ are funded conditionally such that they will be re-evaluated after $q \leq d-1$ periods, i.e., at the beginning of period $t+q \leq t+d-1$. The remaining $\ell-e$ projects are granted full funding for $d$ periods. Based on the re-evaluation, $a$ projects are abandoned, and $e-a$ projects are funded for the remaining duration of $d-q$ periods (for simplicity, we assume that there is no delay between the beginning of the reevaluation process and the decision to abandon/continue projects that have been re-evaluated). The number of projects that will be completed out of those $n$ projects arriving at the beginning of period $t$ is, therefore, $(\ell-e)+(e-a)=\ell-a$. The decision variables $(\ell, e, a, q)$ define the funding policy that is applied to the set of $n$ project proposals arriving in each period. The decision process for projects arriving at the beginning of period $t$ is illustrated in Figure 2.

\section{Insert Figure 2 about here.}

The same decision process is repeated at the beginning of each period $t=$ $1,2, \ldots$ The amount of resources consumed in a given period is thus determined by (i) how many projects have been launched at the beginning of the current and previous periods but not yet re-evaluated, (ii) how many projects have been reevaluated and continued based on the re-evaluation, and (iii) how many projects are to be re-evaluated at the beginning of the current period. Each project, if funded, consumes one resource unit per period, and the cost of re-evaluating a project is $c_{e}$. The resource consumption $C(t)$ in each period $t$ is, therefore,

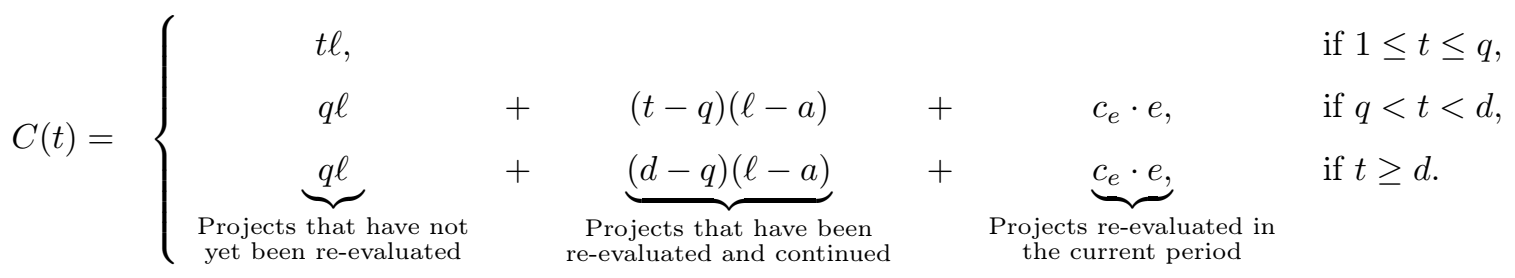


The last line in (1) shows that the period-wise resource consumption of the funding policy over an infinite time horizon stabilizes to $d \ell-(d-q) a+c_{e} e$ after the initial build-up.

We denote the exogenous budget reserved for each funding period by $B$. The set of feasible funding policies is defined as follows.

Definition 1. The set of feasible funding policies is

$\mathcal{P}_{F}=\left\{(\ell, e, a, q) \in \mathbb{N}^{4} \mid d \ell-(d-q) a+c_{e} e \leq B, n \geq \ell \geq e \geq a, 1 \leq q \leq d-1\right\}$.

Definition 1 states that a policy is feasible if (i) the period-wise resource consumption does not exceed the budget $B$, (ii) the number of launched projects does not exceed that of the project proposals, the number of re-evaluated projects does not exceed that of the launched projects, and the number of abandoned projects does not exceed that of the re-evaluated projects, and (iii) projects can be re-evaluated one period after they have been launched at the earliest and one period before completion at the latest.

\subsubsection{Valuation model}

The funding policy $(\ell, e, a, q)$ determines the number of projects that are launched, re-evaluated, and abandoned. Which projects in particular to launch, re-evaluate, and abandon, is based on estimates about the projects' future values. Because we assume that the funding periods are similar a priori (meaning that equally many project proposals arrive in each period and the distributions for the projects' future values and value estimates are the same), we can determine the optimal funding policy by examining the decision process for a single set of $n$ project proposals (such as the one illustrated in Figure 2).

Let us denote by $\left[v_{1}, \ldots, v_{n}\right]$ the future values of the $n$ project proposals such that $v_{i}$ are realizations of independent and identically distributed random variables $V_{i} \sim f(v)$ with a known prior $f(v)$. The future value $v_{i}$ will be realized after project $i$ has been completed. If a project is not completed but abandoned after $q<d$ periods, then the realized salvage value is $h(q) v_{i}$. Here, the function 
$h(q):\{1, \ldots, d-1\} \rightarrow[0,1)$ is assumed to be non-decreasing in $q$, meaning that if the project is abandoned later, then at least as much - and possibly more - value can be retrieved as when abandoning the project earlier. Note that the indirect benefits possibly resulting from exceptional projects are not captured by the future values $v_{i}$.

At the time of the launching decision, the decision-maker does not know the projects' future values but observes estimates $\left[s_{1}^{0}, \ldots, s_{n}^{0}\right]$ thereof. These estimates represent assessments about the future values obtained through quantitative technology forecasting techniques or other methods of technology futures analysis (Porter et al., 2004; Walk, 2012).

The estimates are realizations of conditionally independent and identically distributed random variables $\left(S_{i}^{0} \mid V_{i}=v\right) \sim f\left(s^{0} \mid v\right)$ such that the likelihood distribution $f\left(s^{0} \mid v\right)$ is known for all $v$ and the estimate for project $i$ does not depend on the future values of any other projects. Using the estimates $s_{i}^{0}$ and the distribution assumptions, Bayes' rule $f\left(v \mid s_{i}^{0}\right) \propto f\left(s_{i}^{0} \mid v\right) f(v)$ can be used to compute the posterior distributions $f\left(v \mid s_{i}^{0}\right)$ of the future values $\left(V_{i} \mid S^{0}=s_{i}^{0}\right)$ of the project proposals given the estimates (see Vilkkumaa et al. 2014 for a similar valuation model). The means of these posterior distributions are the projects' expected future values $\mathbb{E}\left[V_{i} \mid S_{i}^{0}=s_{i}^{0}\right]$ given their initial value estimates

$$
\mathbb{E}\left[V_{i} \mid S^{0}=s_{i}^{0}\right]=\int_{-\infty}^{\infty} v f\left(v \mid s_{i}^{0}\right) d v
$$

Let us denote by $s_{i}^{q}$ the interim estimate obtained for project $i$ after $q$ periods. Analogous to $s_{i}^{0}$, these estimates represent the updated assessments about the projects' future values. The interim estimates are realizations of conditionally independent and identically distributed random variables $\left(S_{i}^{q} \mid V_{i}=v\right) \sim f\left(s^{q} \mid v\right)$ with a known distribution function $f\left(s^{q} \mid v\right)$ for all $v$. The posterior distributions $f\left(v \mid s_{i}^{0}, s_{i}^{q}\right)$ for the projects' future values $\left(V_{i} \mid S^{0}=s_{i}^{0}, S^{q}=s_{i}^{q}\right)$ given both the initial and the interim estimates are obtained through Bayes' rule ${ }^{1} f\left(v \mid s_{i}^{0}, s_{i}^{q}\right) \propto$ $f\left(s_{i}^{q} \mid v\right) f\left(v \mid s_{i}^{0}\right)$. Thus, the expected future value of project $i$ given both the initial

\footnotetext{
${ }^{1}$ We assume that given $v, S_{i}^{q}$ is conditionally independent of $S_{i}^{0}$, i.e., $f\left(s_{i}^{q} \mid s_{i}^{0}, v\right)=f\left(s_{i}^{q} \mid v\right)$.
} 
and the interim estimate is

$$
\mathbb{E}\left[V_{i} \mid S_{i}^{0}=s_{i}^{0}, S_{i}^{q}=s_{i}^{q}\right]=\int_{-\infty}^{\infty} v f\left(v \mid s_{i}^{0}, s_{i}^{q}\right) d v
$$

By definition, the future values of exceptional projects are greater than or equal to the excellence threshold $\mu_{\alpha}^{*}$ which is derived from the desired level of excellence $\alpha$ and the prior distribution $f(v)$.

Definition 2. Let $v_{i}$ be a realization of random $V_{i} \sim f(v)$, and let $\mu_{\alpha}^{*}$ be the excellence threshold such that $\mathbb{P}\left(V_{i} \geq \mu_{\alpha}^{*}\right)=\int_{\mu_{\alpha}^{*}}^{\infty} f(v) d v=\alpha$. Project $i$ is an exceptional project if $v_{i} \geq \mu_{\alpha}^{*}$.

The posterior distributions $f\left(v \mid s_{i}^{0}\right)$ and $f\left(v \mid s_{i}^{0}, s_{i}^{q}\right)$ can now be used to compute the probability that project $i$ is an exceptional project given the observed value estimates:

$$
\begin{aligned}
\mathbb{P}\left(V_{i} \geq \mu_{\alpha}^{*} \mid S_{i}^{0}=s_{i}^{0}\right) & =\int_{\mu_{\alpha}^{*}}^{\infty} f\left(v \mid s_{i}^{0}\right) d v \\
\mathbb{P}\left(V_{i} \geq \mu_{\alpha}^{*} \mid S_{i}^{0}=s_{i}^{0}, S_{i}^{q}=s_{i}^{q}\right) & =\int_{\mu_{\alpha}^{*}}^{\infty} f\left(v \mid s_{i}^{0}, s_{i}^{q}\right) d v .
\end{aligned}
$$

All decision variables and model parameters are listed in Table 1.

\subsubsection{Optimal funding policies}

We next determine the optimal funding policies $(\ell, e, a, q)$. Toward this end, 325 let us denote by $\mathcal{N}=\{1, \ldots, n\}$ the index set of all new project proposals, by $\mathcal{L} \subseteq \mathcal{N}$ the index set of launched projects, by $\mathcal{E} \subseteq \mathcal{L}$ the index set of those launched projects that are to be re-evaluated, and by $\mathcal{A} \subseteq \mathcal{E}$ the index set of the projects that are abandoned based on the re-evaluation. These index sets correspond to funding policy $(\ell, e, a, q)$ through $|\mathcal{L}|=\ell,|\mathcal{E}|=e$, and $|\mathcal{A}|=a$ (where, for instance, $|\mathcal{L}|$ denotes the number of elements in the set $\mathcal{L}$ ). The optimal index sets depend on not only the expectations about the projects' future values but also the decision objective.

First, we consider the objective of maximizing the expected portfolio value. Based on the independence assumptions, the expected value of the project portfolio is the sum of the expected values of the projects it includes. The funding 
Table 1: List of decision variables and model parameters

\begin{tabular}{ll}
\hline Decision variables & \\
\hline$\ell$ & Number of projects launched at the beginning of each period \\
$e$ & Number of projects re-evaluated at the beginning of each period \\
$a$ & Number of projects abandoned at the beginning of each period \\
$q$ & Number of periods after which projects are re-evaluated \\
\hline Parameters & \\
\hline$n$ & Number of new project proposals in each period \\
$d$ & Duration of each project \\
1 & Cost of funding one project for one period (=1) \\
$c_{e}$ & Cost of re-evaluating one project \\
$B$ & Budget for each period \\
$\alpha$ & Level of excellence \\
$f(v)$ & Prior distribution of the projects' future values \\
$f\left(s^{0} \mid v\right)$ & Distribution of a project's initial value estimate given its future value \\
$f\left(s^{q} \mid v\right)$ & Distribution of a project's interim value estimate obtained $q$ periods \\
& after launch, given its future value \\
$h(q)$ & Non-decreasing function with range [0,1) which determines the fraction of \\
& the project's future value that can be salvaged if the project is abandoned \\
& $q$ periods after launch \\
\hline
\end{tabular}

policy that maximizes the expected portfolio value can, therefore, be determined by solving the two-stage stochastic optimization problem

$\max _{(\ell, e, a, q) \in \mathcal{P}_{F}} \mathbb{E}_{S_{i}^{0}}\left[\max _{\substack{\mathcal{L} \subseteq \mathcal{\mathcal { L }}, \mathcal{E} \subseteq \mathcal{L} \\|\mathcal{L}|=\ell \\|\mathcal{E}|=e}}\left\{\sum_{i \in \mathcal{L} \backslash \mathcal{E}} \mathbb{E}\left[V_{i} \mid S_{i}^{0}\right]+\mathbb{E}_{S_{i}^{q}}\left[\max _{\substack{\mathcal{A} \subseteq \mathcal{E} \\|\mathcal{A}|=a}}\left\{\sum_{i \in \mathcal{E} \backslash \mathcal{A}} \mathbb{E}\left[V_{i} \mid S_{i}^{0}, S_{i}^{q}\right]+h(q) \sum_{i \in \mathcal{A}} \mathbb{E}\left[V_{i} \mid S_{i}^{0}, S_{i}^{q}\right]\right\}\right]\right\}\right]$,

where set of feasible policies $\mathcal{P}_{F}$ is as in Definition 1 , and the expected values $\mathbb{E}\left[V_{i} \mid S_{i}^{0}\right]$ and $\mathbb{E}\left[V_{i} \mid S_{i}^{0}, S_{i}^{q}\right]$ are obtained from (3) and (4) with random $S_{i}^{0}$ and $335 S_{i}^{q}$.

The optimization is carried out in two stages: in the first stage with regard to the funding policy $(\ell, e, a, q)$, and in the second stage with regard to the index sets $\mathcal{L}, \mathcal{E}$, and $\mathcal{A}$. The maximization in the second stage is further divided into 
two parts: (i) the determination of the sets $\mathcal{L}$ and $\mathcal{E}$ of launched and re-evaluated 340 projects based on the initial estimates only and (ii) the determination of the set $\mathcal{A}$ of abandoned projects based on both the initial and interim estimates. Given the funding policy $(\ell, e, a, q)$, the cardinalities of these index sets remain constant.

Second, we consider the objective of maximizing the number of completed projects that qualify as exceptional projects. Given the excellence threshold $\mu_{\alpha}^{*}$, the policy that maximizes this number is the solution to the two-stage stochastic optimization problem

$\max _{(\ell, e, a, q) \in \mathcal{P}_{F}} \mathbb{E}_{S_{i}^{0}}\left[\max _{\substack{\mathcal{L} \subseteq \mathcal{N}, \mathcal{E} \subseteq \mathcal{L} \subseteq \mathcal{L} \\|\mathcal{L}|=\ell \\|\mathcal{E}|=e}}\left\{\sum_{i \in \mathcal{L} \backslash \mathcal{E}} \mathbb{P}\left(V_{i} \geq \mu_{\alpha}^{*} \mid S_{i}^{0}\right)+\mathbb{E}_{S_{i}^{q}}\left[\max _{\substack{\mathcal{A} \subseteq \mathcal{E} \\|\mathcal{A}|=a}} \sum_{i \in \mathcal{E} \backslash \mathcal{A}} \mathbb{P}\left(V_{i} \geq \mu_{\alpha}^{*} \mid S_{i}^{0}, S_{i}^{q}\right)\right]\right\}\right]$,

where the probabilities $\mathbb{P}\left(V_{i} \geq \mu_{\alpha}^{*} \mid S_{i}^{0}\right)$ and $\mathbb{P}\left(V_{i} \geq \mu_{\alpha}^{*} \mid S_{i}^{0}, S_{i}^{q}\right)$ are obtained from (5) and (6) with random $S_{i}^{0}$ and $S_{i}^{q}$.

The objective function in (8) does not include abandoned projects because only completed projects are assumed to qualify as exceptional projects. The optimization is again carried out in two stages. The first term in the secondstage objective function of (8) sums the probabilities that the projects that have received full funding based on initial evaluations are indeed exceptional projects. The second term sums the probabilities that those projects that have been reevaluated and eventually completed are exceptional projects. Because we have assumed that the projects' future values are independent and that the value estimate of project $i$ does not depend on the future values of other projects, 355 the objective function of (8) gives the expected number of funded (completed) exceptional projects.

The value of the abandonment option is defined as the relative improvement in the portfolio value with the option compared to the portfolio value without the option, i.e.,

Option value $=\frac{\text { Portfolio value with option }- \text { Portfolio value without option }}{\text { Portfolio value without option }}$.

Here, portfolio value with the option is the optimal value of the objective func- 
tion in (7) or (8), and portfolio value without the option is obtained by setting $e=a \equiv 0$ in (7) or (8).

\section{Results}

\subsection{Parameters}

The above model allows us to establish guidelines for funding policies that yield either the highest expected value or the highest expected number of exceptional projects over time. Because no analytical solutions can be derived

365 for problems (7) and (8), these guidelines are obtained by numerical simulation. In particular, we study how the optimal funding policy and the resulting option value depend on the problem parameters when both the prior and the likelihood distributions (and, therefore, the posterior distributions as well) are normal. The normal distribution is commonly used for modeling the randomness of many types of real-world phenomena. For instance, due to the central limit theorem, the use of a normal likelihood is well justified if the value estimates are averages over the evaluations of unbiased experts with equal evaluation accuracies. Thus, the qualitative insights provided by this case are informative even if the underlying distributions are not exactly normal.

We assume that the projects' future values are realizations of independent and identically distributed random variables $V_{i} \sim \mathrm{N}\left(20,3^{2}\right)$. The initial estimates are obtained from the future values by an additive, normally distributed zero-mean estimation error term such that $\left(S_{i}^{0} \mid V_{i}=v_{i}\right)=v_{i}+\delta^{0}, \delta^{0} \sim \mathrm{N}\left(0, \tau_{0}^{2}\right)$ for each $i=1, \ldots, n$. The interim estimates are modeled similarly such that

38о $\left(S_{i}^{q} \mid V_{i}=v_{i}\right)=v_{i}+\delta^{q}, \delta^{q} \sim \mathrm{N}\left(0, \tau_{q}^{2}\right)$ for each $i=1, \ldots, n$. Typically, the accuracy of the estimates improves significantly in time (Walk, 2012). We model this improvement by relating the standard deviation $\tau_{q}$ of the interim estimate to the initial estimation error through $\tau_{q}=r^{q} \tau_{0}$, where $r \in(0,1]$. This model for the expected evolution of the standard deviation of the estimation error results in a cone of uncertainty (Boehm, 1981), where initially very little may be known about the future value of a project, but as time goes by, the uncertainty about 
this value reduces geometrically. The rate at which the uncertainty diminishes is determined by the uncertainty reduction coefficient $r$.

We consider $n=200$ new project proposals in each period. The duration of each project is $d=4$, the cost of funding one project for one period is 1 , and the cost of re-evaluating one project is $c_{e}=0.05$. The budget $B$ is set so that it would correspond to providing full funding to $30 \%$ of the project proposals in each period if no resources were spent on acquiring interim evaluations, i.e., $B=0.3 \times n \times 1 \times d=240$. The salvage value is assumed to be $h(q) \equiv 0$, so that no value can be retrieved from abandoned projects. We examine how both the optimal funding policy and the option value change as a function of the following parameters:
$\tau_{0}: \quad$ Standard deviation of the initial value estimates,
$r$ : Coefficient of uncertainty reduction,
$\alpha: \quad$ Level of excellence.

Sensitivity analyses with respect to changes in $n, B$, and $h(q)$ are performed in Section 4.6.

\subsection{Computation of the optimal funding policies}

Problems (7) and (8) can be solved by simulating the decision process for each feasible policy multiple (e.g., 5,000) times, and then selecting the policy that, on average, yields the highest value or the highest number of exceptional projects. For fixed $(\ell, e, a, q)$, the decision process is simulated by first sampling future values $v_{i}$ and value estimates $s_{i}^{0}, s_{i}^{q}$ from the prior and likelihood distributions $f(v), f\left(s^{0} \mid v\right)$, and $f\left(s^{q} \mid v\right)$. Then, the objective function (7) or (8) is evaluated for all possible combinations $\mathcal{L}, \mathcal{E}, \mathcal{A}$ corresponding to the fixed funding policy, and the highest objective function value in that simulation round (i.e., given the particular realization of $v$ ) is recorded. These values are then averaged over all simulation rounds to obtain the average objective function values for the fixed funding policy. By repeating this process for each feasible funding policy $(\ell, e, a, q)$, one can find the policies that yield the highest aver- 
age portfolio value or the highest average number of exceptional projects in the 405 portfolio, i.e., the solutions to problems (7) and (8).

The computation effort can be reduced by the following two propositions. All proofs are in the Appendix.

Proposition 1. Assume $f(v)=\mathrm{N}\left(\mu, \sigma^{2}\right), f\left(s^{0} \mid v\right)=\mathrm{N}\left(v, \tau_{0}^{2}\right)$, and $f\left(s^{q} \mid v\right)=$ $\mathrm{N}\left(v, \tau_{q}^{2}\right)$. Then, given a fixed funding policy $(\ell, e, a, q)$ and estimates $s_{i}^{0}$ and ${ }_{410} s_{i}^{q}$, the optimal index sets for the second-stage optimization problem in both (7) and (8) are

$$
\begin{aligned}
& \mathcal{A}=\left\{i \in \mathcal{E} \mid v_{i}^{q} \text { among the a smallest }\right\} \\
& \mathcal{E}=\left\{i \in \mathcal{L} \mid v_{i}^{0} \text { among the } e \text { smallest }\right\} \\
& \mathcal{L}=\left\{i \in \mathcal{N} \mid v_{i}^{0} \text { among the } \ell \text { largest }\right\}
\end{aligned}
$$

where $v_{i}^{0}=\mathbb{E}\left[V_{i} \mid S_{i}^{0}=s_{i}^{0}\right]$ and $v_{i}^{q}=\mathbb{E}\left[V_{i} \mid S_{i}^{0}=s_{i}^{0}, S_{i}^{q}=s_{i}^{q}\right]$.

Proposition 1 states that it is optimal to launch those projects with the highest initial expected values, to re-evaluate those among the launched projects 415 with the lowest initial expected values, and to abandon those with the lowest expected values given also the interim value estimates. Thus, the funding policy $(\ell, e, a, q)$ determines the optimal index sets $\mathcal{L}, \mathcal{E}, \mathcal{A}$ completely, which considerably speeds up the computation. Without this property, we would have to enumerate all $\left(\begin{array}{l}n \\ \ell\end{array}\right)\left(\begin{array}{l}\ell \\ e\end{array}\right)\left(\begin{array}{l}e \\ a\end{array}\right)$ combinations of $\mathcal{L}, \mathcal{E}$ and $\mathcal{A}$ for each choice of $\ell, e$ 420 and $a$. If, for instance, $n=10, \ell=6, e=4$ and $a=2$, the number of such combinations would be 18,900 .

The computation time is further reduced by Proposition 2, which states that in the optimum, no additional projects can be re-evaluated if all other policy variables remain the same. Given that $(\ell, a, q)$ are fixed, the optimal value of $e$ 425 is, thus, $e_{\max }=\min \left\{\ell,\left\lfloor\frac{1}{c_{e}}(B-d \ell+(d-q) a)\right\rfloor\right\}$. It is therefore not necessary to compute the value for any policy $(\ell, e, a, q)$ where $e<e_{\max }$. If, for instance, $\ell=6, a=2, q=1, B=13$ and $c_{e}=0.2$, we have $e_{\max }=\min \{6,5\}=5$, implying that it is unnecessary to compute the value of policies in which $e=2,3$, or 4. Using Propositions 1 and 2, the total computation time for all numerical 
results in this paper was approximately two and a half hours using Matlab on a standard laptop (2.60 GHz, 8 GB memory).

Proposition 2. If policy $(\ell, e, a, q)$ is optimal for either (7) or (8), then policy $(\ell, e+1, a, q)$ is infeasible.

\subsection{Features of the optimal funding policy}

435 4.3.1. Estimation uncertainty $\tau_{0}$

Figure 3 illustrates the optimal funding policy for either accurate $\left(\tau_{0}=2\right)$ or inaccurate $\left(\tau_{0}=7\right)$ initial estimates, when the objective is to either maximize the expected portfolio value (Figures $3 \mathrm{a}$ and $3 \mathrm{~b}$ ), or to maximize the expected number of funded projects in the top 1\% (Figures 3c and 3d). The uncertainty reduction coefficient is set at $r=0.5$, meaning that in each period, the standard deviation of the estimation error decreases by $50 \%$. The figures use the following notation:

$R: \quad \quad \quad$ of projects that are rejected completely $(n-\ell)$,

$C F: \quad \quad \quad \quad$ of projects that receive conditional funding and will be re-evaluated $(e)$,

$F F: \quad \quad \quad \quad$ of projects that receive full funding for their entire duration $(\ell-e)$,

$C: \quad \quad \quad$ of projects that are continued based on the re-evaluation $(e-a)$,

$A$ : $\quad$ \# of projects that are abandoned based on the re-evaluation $(a)$.

Each figure profiles the decisions only for those projects launched at the beginning of period 1, thus omitting - for purposes of clarity - the presence of other projects launched at the beginning of periods 2,3 , and 4 .

\section{Insert Figure 3 about here.}

Regardless of the accuracy of the initial estimates, the policy that maximizes the expected portfolio value is simply to grant full funding to the 60 projects with the highest initial expected values. This policy differs from the policy that maximizes the number of exceptional projects, which is to launch a large number of projects $(\ell=F F+C F)$, re-evaluate most of them $(e=C F)$ - in 
fact, all of them, if the initial estimates are inaccurate -, and abandon a large share of the re-evaluated projects. When the initial estimates are more accurate, less resources are spent on project evaluation, which allows more projects to be completed $(\ell-a=F F+C)$.

\subsubsection{Uncertainty reduction coefficient $\mathbf{r}$ and level of excellence $\boldsymbol{\alpha}$}

Figure 4 illustrates the optimal funding policy for fast $(r=0.4)$ and slow $(r=0.8)$ rates of uncertainty reduction when the objective is to maximize the number of funded projects whose future values are either in the top $\alpha=10 \%$ or top $\alpha=1 \%$ of the prior distribution. Here, the standard deviation of the initial estimation error is set at $\tau_{0}=7$.

If the estimation uncertainty reduces quickly, meaning that the interim estimates are much more accurate than the initial ones (Figures 4a and 4c), then more projects should be launched and abandoned. The more exceptional the projects the decision-maker wishes to fund (i.e., the smaller $\alpha$ ), the larger these shares are. On the other hand, if the interim estimates are not significantly more accurate, then it is optimal to allocate most resources to completing projects and to spend less resources on evaluation (Figures $4 \mathrm{~b}$ and $4 \mathrm{~d}$ ). In this case the optimal policy is more like that in Figures 3a and 3b.

Figure 4 shows - perhaps counterintuitively - that the decision-maker should wait longer before abandoning projects when the accuracy of estimates improves more quickly: in Figures 4a and 4c projects are abandoned after $q=2$ periods, whereas in Figures $4 \mathrm{~b}$ and $4 \mathrm{~d}$ the decision to abandon projects is made after just ${ }_{475} q=1$ period. This reflects the trade-off between the number of projects that can be completed and the amount of time the decision-maker can wait for the estimates to become sufficiently accurate to justify the abandonment of many projects. With $r=0.8$ (Figures $4 \mathrm{~b}$ and $4 \mathrm{~d}$ ), this takes too much time, whereby resources should mostly be spent on the execution of projects. 
Results for the optimal abandonment time $q$ when maximizing the expected number of projects in the top $1 \%$ based on the objective function in (8) are shown in Figure 5 as a function of the uncertainty reduction coefficient $r$. In Figure 5a the initial estimates are moderately inaccurate $\left(\tau_{0}=6\right)$, and in Fig${ }_{485}$ ure $5 \mathrm{~b}$ very inaccurate $\left(\tau_{0}=13\right)$. For reference, the optimal policy for maximizing the expected portfolio value based on the objective function in (7) for each combination of $r$ and $\tau_{0}$ in this setting is not to exercise the option.

Insert Figure 5 about here.

In general, one should wait longer before abandoning projects when the 490 initial estimates are less accurate. Moreover, Figure 5 shows that the optimal abandonment time may first increase and then decrease in $r$. This reflects the trade-off between allocating resources either to completing more projects or to obtaining more accurate evaluations through experimentation. For instance, Figure 5a illustrates that when $r \leq 0.4$, the estimation uncertainty reduces very fast, whereby the interim evaluations become sufficiently accurate already after one period to make decisions about abandoning projects. When $0.5 \leq r \leq 0.6$, the accuracy in the estimates improves more slowly, and, hence, the decisionmaker should re-evaluate the projects only after two periods. Finally, when $r \geq$ 0.7 , the interim estimates remain inaccurate throughout the projects' duration, whereby resources are optimally spent on completing more projects rather than launching many projects and abandoning some of them based on more accurate, but still unreliable, information.

A similar non-monotonic dependence between the expected time-to-decision and the uncertainty in a project's profit is discovered by Kwon and Lippman ${ }_{505}$ (2011). Based on a project's observed profit stream, they use a Bayesian framework to update the probability that the drift of the project's profit is either at a high or a low state. In this setting, they note that the expected optimal time to either abandon or expand the project first increases and then decreases in 
the volatility of the profit stream.

5104.4 . Characteristics of the option value

\subsubsection{Estimation uncertainty $\boldsymbol{\tau}_{\mathbf{0}}$ and uncertainty reduction coefficient $\mathbf{r}$}

Figure 6 shows the option value as a function of the standard deviation $\tau_{0} \in$ $\{0,1, \ldots, 10\}$ of the initial estimation error for different values of the uncertainty reduction coefficient $r$, when the objective is to maximize the number of funded

515 projects among the top 1\%. The figure also shows the optimal abandonment times $q$ corresponding to each combination of $r$ and $\tau_{0}$. Again, the optimal policy for maximizing the expected portfolio value for each $r$ and $\tau_{0}$ in this setting is not to exercise the option, so that the corresponding option value is zero.

The option value decreases in $r$ for all $\tau_{0}$, meaning that the option is more valuable when the interim estimates are relatively more accurate. However, all three curves corresponding to different values of $r$ first increase and then start to decrease after $\tau_{0}$ reaches a certain point. The non-monotonic dependence of the option value on $\tau_{0}$ stems from the fact that for low values of $\tau_{0}$, the initial decision is based on accurate information, and, hence, the option value is small. On the other hand, if $\tau_{0}$ is very large, the interim estimates are also quite unreliable since $\tau_{q}=r^{q} \tau_{0}$, and consequently the option value is small. At the peak point, the value gained from reducing the standard deviation of the estimation error by factor $r^{q}$ is maximal. Here, in particular, the highest option value ( $26 \%$ increase in the number of exceptional projects in the portfolio compared to not having the option) is attained when the initial estimates are very inaccurate $\left(\tau_{0}=9\right)$, given that more accurate estimates can be obtained relatively quickly $(r=0.4)$.

${ }_{535}$ A similar non-monotonic relationship between uncertainty and option value has been discovered by Siddiqui and Fleten (2010) who study the value of the 
option for an intermediate learning stage between switching from an existing renewable energy technology to an unconventional technology with uncertain operating cost, the expected value of which can be reduced during this learning stage. They show that for low rates of operating cost reduction, the option value of the learning stage first increases and then decreases in the long-term electricity price volatility.

\subsubsection{Estimation uncertainty $\tau_{\mathbf{0}}$ and level of excellence $\boldsymbol{\alpha}$}

Figure 7 illustrates the option value when maximizing the number of excep545 tional projects in the portfolio as a function of the initial estimation uncertainty $\tau_{0}$ for different levels of excellence $\alpha$. In Figure 7a, the estimation uncertainty reduces quickly with $r=0.4$, and in Figure $7 \mathrm{~b}$ it reduces slowly with $r=0.8$. As noted before, the option value first increases and then decreases in the initial estimation uncertainty $\tau_{0}$ and is higher when the uncertainty reduces more 550 quickly.

Insert Figure 7 about here.

Interestingly, given that the initial estimates are accurate, the option value is lower when the decision-maker is interested in truly exceptional projects, i.e., those with very high future values $(\alpha=1 \%)$. This is because when the initial estimates are accurate, a large share of the top $1 \%$ projects are funded just by completing a sufficiently large number of projects, whereby the option value is small. On the other hand, when the initial estimates are very inaccurate, the option is more valuable when the decision-maker is interested in projects in the top $1 \%$. This is due to the fact that it is not necessary to complete many projects to fund those in the top $1 \%$, given that the information based on which the completed projects are selected is very accurate. Thus, it can be very valuable to experiment on a large number of projects, obtain relatively accurate estimates about these projects later on and, based on these estimates, commit resources to only those few projects that are likely to be among the top $1 \%$. 
Based on Figure 7, the abandonment option has the most value (26\%) when the initial value estimates are very inaccurate $\left(\tau_{0}=9\right)$, the accuracy improves quickly $(r=0.4)$, and the decision-maker is interested in truly exceptional projects $(\alpha=1 \%)$. On the other hand, if the initial estimates are very inaccurate and the accuracy improves slowly, then the option has little value. In this case, the interim evaluations provide poor justification for abandoning projects, and, hence, it pays off to use most resources to completing as many projects as possible, i.e., to provide full funding to those projects that appear to yield the most value based on the initial estimates.

\subsection{Cross-comparison of funding policies}

Next, we compare how the optimal policy for maximizing the expected number of exceptional projects performs in terms of expected portfolio value, and vice versa. Here, the initial estimation uncertainty is $\tau_{0}=8$, the uncertainty reduction coefficient is $r=0.3$, and the level of excellence is $\alpha=1 \%$. Table 2 shows the number of completed projects, the expected portfolio value, the expected average project value (= the expected portfolio value / the number of completed projects), and the expected number of exceptional projects in the portfolio for two policies:

- Policy 1 (optimal for the maximization of the expected portfolio value):

- Full funding for 60 out of 200 project proposals in each period.

585 - Policy 2 (optimal for the maximization of the expected number of funded projects whose future values are in the top $1 \%$ ):

- Conditional funding for 108 out of 200 project proposals in each period,

- All projects re-evaluated after two periods,

- Out of the re-evaluated projects, 99 abandoned and 9 completed. 
Table 2: Cross-comparison of funding policies with $\tau_{0}=8$ and $r=0.3$.

\begin{tabular}{lcc}
\multicolumn{3}{c}{ Table 2: Cross-comparison of funding policies with $\tau_{0}=8$ and $r=0.3}$. \\
\hline Policy 1 & Policy 2 \\
\hline Number of completed projects & 60 & 9 \\
Expected portfolio value & 1,273 & 233 \\
Expected average project value & 21.22 & 25.86 \\
Expected number of exceptional projects & 1.28 & 1.68
\end{tabular}

Table 2 illustrates that the average value of funded projects is higher when adopting policy 2, which is optimal for funding exceptional projects (25.86 vs. 21.22). Furthermore, given that the expected number of exceptional projects in the population is $n \cdot \alpha=200 \cdot 1 \%=2$, the adoption of policy 2 results in funding $1.68 / 2=84 \%$ of such projects, whereas only $1.28 / 2=64 \%$ of them would be funded if policy 1 was adopted. However, due to completing only 9 projects out of 200 proposals in each period - as opposed to 60 completed projects out of 200 resulting from policy 1 - the expected portfolio value of policy 2 is much lower than that of policy 1 (233 vs. 1,273). Thus, if the potential for extremely high indirect benefits of exceptional projects is not accounted for, then policies that promote such projects may appear cost-inefficient in the short term. Conversely, funding policies that are cost-efficient in the short term may fail to pick those projects that could eventually yield extremely high societal benefits.

Figure 8 illustrates the impact of adopting the 'wrong' policy as a function 605 of the initial estimation uncertainty for fast $(r=0.3)$, moderate $(r=0.65)$, and slow $(r=0.9)$ rates of uncertainty reduction. In particular, Figure 8a shows the percentage of the expected portfolio value obtained by adopting a policy that maximizes the expected number of exceptional projects compared to what could have been obtained by adopting a policy that maximizes this value. The optimal abandonment times for the policies maximizing the expected number of exceptional projects are also shown.

Insert Figure 8 about here. 
If the initial estimates are perfectly accurate $\left(\tau_{0}=0\right)$, then the optimal policy is the same for both objectives, i.e., to simply select the best 60 projects out 615 of the 200 proposals. On the other hand, the less accurate the initial estimates and the slower the accuracy is improved, the more similar the optimal policies are for maximizing the expected portfolio value and the expected number of exceptional projects. Thus, there is not much difference in the portfolio values in Figure 8a when, for instance, $r=0.9$ and $\tau_{0} \geq 7$. However, if the initial estimates are relatively accurate and/or the estimation accuracy improves quickly, then resources are optimally spent on launching many but completing only a few projects. In this case, the expected portfolio value resulting from the maximization of the number of exceptional projects is much lower compared to when maximizing this value; especially so if one should wait for two periods instead

${ }_{625}$ of one before abandoning projects, which is the case when $\tau_{0} \geq 8$ for $r=0.3$, and $\tau_{0} \geq 6$ for $r=0.65$.

Figure $8 \mathrm{~b}$ illustrates the percentage of the number of exceptional projects funded by the policy that maximizes the expected portfolio value compared to the policy that maximizes the expected number of exceptional projects. Here, the policy that maximizes the expected portfolio value for each $\tau_{0}$ and $r$ is to not exercise the option, whereby no optimal abandonment times are shown. When the initial estimates are very accurate $\left(\tau_{0} \leq 2\right)$, most of the top $1 \%$ projects are funded just by providing full funding for the best projects based on the initial evaluation, whereby the difference between the shares of exceptional projects resulting from the two policies is small. This difference remains small even for larger values of $\tau_{0}$ when the uncertainty reduces slowly $(r=0.9)$, because in this case the optimal policies are not that different. However, if the initial estimates are very inaccurate $\left(\tau_{0}=10\right)$ - as they typically are - but more accurate estimates can be obtained later on $(r=0.3)$, then the failure to use such estimates ${ }_{640}$ to abandon projects and, thus, to release resources for launching more projects would result in funding only $75 \%$ of those exceptional projects that would be funded by following the optimal strategy for funding exceptional projects. Because of the potential for extremely high indirect benefits of exceptional projects, 
such a difference is significant.

645 4.6. Sensitivity analysis

Finally, we present results on the impact of the variation in parameters $n$ and $B$ as well as in the shape of the salvage value function $h(q)$. Table 3 shows the optimal funding policies with respect to the two objectives for different values of $n$ such that the initial estimation uncertainty $\tau_{0}=7$ is reduced by $50 \%$ in each period, i.e., $r=0.5$. The level of excellence is $\alpha=1 \%$, the budget is $1.2 n$, and the salvage value function is linear, $h(q)=q / d$. For $n \leq 50$, the optimal policy for maximizing the expected portfolio value is to provide full funding to all launched projects, whereas for larger $n$ it pays off to re-evaluate more than half of the launched projects and abandon quite a few. This is because the re-evaluation of $e$ projects out of a smaller number $n$ of proposals would require a comparatively larger fraction of resources, as the budget $B=1.2 n$ is defined in proportion to $n$. Even though some value can now be retrieved from abandoned projects, it is still optimal for all $n$ to launch, re-evaluate, and abandon a larger share of projects when maximizing the number of exceptional projects than when maximizing the expected portfolio value; which is in line with the results presented in this section.

\begin{tabular}{c|cccc|cccc}
\multicolumn{1}{c}{ Table 3: Optimal funding policies for different values of $n}$. \\
& \multicolumn{3}{|c}{ Expected value } & \multicolumn{5}{c}{ Exceptional projects } \\
& $\frac{\ell}{n}$ & $\frac{e}{n}$ & $\frac{a}{n}$ & $q$ & $\frac{\ell}{n}$ & $\frac{e}{n}$ & $\frac{a}{n}$ & $q$ \\
\hline$n=10$ & $30 \%$ & 0 & 0 & - & $30 \%$ & $30 \%$ & $10 \%$ & 2 \\
$n=50$ & $30 \%$ & 0 & 0 & - & $42 \%$ & $42 \%$ & $26 \%$ & 2 \\
$n=200$ & $37 \%$ & $20 \%$ & $9 \%$ & 1 & $48 \%$ & $48 \%$ & $37 \%$ & 2 \\
$n=500$ & $38 \%$ & $24 \%$ & $12 \%$ & 1 & $48 \%$ & $48 \%$ & $38 \%$ & 2
\end{tabular}

Table 4 illustrates the impact of size of the budget $B$ on the optimal funding policies. Here, $n=200$, and the salvage value function is linear, $h(q)=q / d$. Budget 120 corresponds to providing full funding for $B /(n \cdot d)=120 /(200 \cdot 4)=$ $66515 \%$ of the project proposals, budget 240 to $30 \%$, and budget 320 to $40 \%$. 
The larger the budget, the more projects can be launched, re-evaluated, and completed $\left(\frac{\ell}{n}-\frac{a}{n}\right)$. Again, for all values of $B$, it is optimal to launch, reevaluate, and abandon a larger share of projects when maximizing the expected number of exceptional projects than when maximizing the expected portfolio value.

\begin{tabular}{c|ccccc|ccccc} 
& \multicolumn{8}{|c}{ Table 4: Optimal funding policies for different values of $B}$. \\
& \multicolumn{7}{c}{ Expected value } & \multicolumn{7}{c}{ Exceptional projects } \\
& $\frac{\ell}{n}$ & $\frac{e}{n}$ & $\frac{a}{n}$ & $\frac{\ell}{n}-\frac{a}{n}$ & $q$ & $\frac{\ell}{n}$ & $\frac{e}{n}$ & $\frac{a}{n}$ & $\frac{\ell}{n}-\frac{a}{n}$ & $q$ \\
\hline$B=120$ & $19 \%$ & $10 \%$ & $6 \%$ & $13 \%$ & 1 & $22 \%$ & $22 \%$ & $15 \%$ & $7 \%$ & 2 \\
$B=240$ & $37 \%$ & $20 \%$ & $9 \%$ & $28 \%$ & 1 & $48 \%$ & $48 \%$ & $37 \%$ & $11 \%$ & 2 \\
$B=320$ & $49 \%$ & $40 \%$ & $18 \%$ & $31 \%$ & 2 & $64 \%$ & $64 \%$ & $49 \%$ & $15 \%$ & 2
\end{tabular}

Finally, Table 5 illustrates the optimal policies for different shapes of the salvage value function $h(q)$ : constant zero, convex, linear, concave and $S$-shaped (see Figure 9). The constant zero $h(q)$ describes, for instance, sequential technology development projects (Roberts and Weitzman, 1981) in which all stages ${ }_{675}$ must be completed to receive any benefits. The $S$-shaped salvage value function, in turn, is widely used for modeling the performance improvement of technologies over their lifetimes, whereas the convex salvage value function describes sharply increasing performance curves such as the improvement in microprocessor transistor density over time (Schilling, 2008). The linear salvage value function has been criticized for decades for failing to describe project development realistically, especially when little is initially known about the project's future value (Godin, 2005); indeed, the linear $h(q)$ as well as the rarely used concave $h(q)$ are included in this analysis for the sake of reference.

Insert Figure 9 about here.

Here, $n=200$, and the budget is $B=240$. Because the objective function for maximizing the expected number of exceptional projects does not depend on the salvage value function, the optimal policy for this objective is the same 
for all choices of $h(q)$, namely $(95,95,73,2)=(48 \%, 48 \%, 37 \%, 2)$. When $h(q)$ in constant zero or convex, the optimal policy for maximizing the expected portfolio value is to give full funding to all launched projects. This is because a large share of a project's value can be obtained only if the project is completed - in fact, all of it, if $h(q)$ is constant zero -, whereby it does not pay off to abandon projects prematurely.

Table 5: Funding policies that maximize the expected portfolio value for different salvage value functions $h(q)$.

\begin{tabular}{l|cccc} 
& $\ell$ & $e$ & $a$ & $q$ \\
\hline$h(q) \equiv 0$ & $30 \%$ & 0 & 0 & - \\
$h(q)$ convex & $30 \%$ & 0 & 0 & - \\
$h(q)$ linear & $37 \%$ & $20 \%$ & $9 \%$ & 1 \\
$h(q)$ concave & $100 \%$ & $100 \%$ & $95 \%$ & 1 \\
$h(q) S$-shaped & $39 \%$ & $39 \%$ & $38 \%$ & 3
\end{tabular}

The above conclusion does not, however, hold when $h(q)$ is concave or $S$ shaped. If the salvage value function is concave, then the expected portfolio value would be maximized by launching all 200 projects but abandoning 190 (i.e., 95\%) of them after just one period. It may not, however, be very realistic to assume that almost half of the projects' benefits would be obtained after only a quarter of the projects' duration (Figure 9). Also, if the function $h(q)$ is $S$-shaped, then it would be optimal to abandon nearly all launched projects just one period before completion. By doing so, one would avoid the costs of funding projects in the last period, when little additional value is generated. This kind of a situation could, however, be interpreted such that the projects are essentially completed at the time of abandonment. Hence, the optimal policy would actually correspond to providing full funding for all launched projects, which is consistent with the results reported in this section. 


\section{Discussion and Conclusions}

Breakthrough technologies that yield extremely high benefits for society are very rare. Hence, the fostering of breakthrough technologies is typically pursued 710 by trying to fund exceptionally valuable technology projects that have the potential to result in breakthroughs. Our results suggest that if the objective is to maximize the expected number of exceptional projects, the optimal policy is to experiment on a large number of projects for some time and, based on this experimentation, commit resources only to those few projects which appear best.

715 This policy differs from the policy of maximizing expected portfolio value, which is to provide full funding to those projects that appear best based on the initial value estimates. These differences are important in that a policy which serves to maximize the expected portfolio value may fail to promote breakthrough technologies. Conversely, a policy which serves to promote breakthrough technologies may result in lower average short-term portfolio value, because many ongoing projects will be discontinued before completion.

The abandonment option involves a trade-off between allocating resources to (i) the completion of on-going projects and (ii) the acquisition of more accurate evaluations to support interim project abandonment decisions. In the absence of interim evaluations, resources will be tied to projects for their entire duration and consequently fewer new projects can be launched, meaning that there is a risk of failing to launch new projects that could have resulted in a breakthrough. Still, if more projects are launched and these projects are funded longer, it follows that more projects will have to be abandoned due to the scarcity of resources. In fact, the risk of missing out on breakthrough technologies is partly decreased by the ability to select which projects should be completed based on more accurate value information, but partly increased by the fact that fewer projects can be completed.

In our model, the trade-off between the number of completed projects and the acquisition of more accurate project evaluations is explicit, as is the dependence of this trade-off on the accuracy of the initial evaluations and the 
rate at which this accuracy is improved through experimentation. In particular, our results suggest that the more uncertain the initial evaluations, the more resources should be devoted to acquiring value information through (i) experimenting longer, if the evaluation accuracy improves slowly, or (ii) launching and abandoning more projects, if the evaluation accuracy improves quickly.

The rate at which the evaluation accuracy improves depends on several factors, such as the nature of the technological field or the phase of the innovation process. Nevertheless, in some cases both the initial evaluation accuracy and rate at which the accuracy is improved can be affected by allocating more resources to project evaluation through obtaining more evaluations of each project or using more competent evaluators, for instance. Our model could be extended to support such resource allocation decisions by (i) treating the evaluation cost $c_{e}$ as a decision variable instead of a constant, and (ii) modeling the standard deviation $\tau_{0}$ of the initial estimation error and the uncertainty reduction coefficient $r$ as decreasing functions of $c_{e}$. Other model parameters, too, can be used to capture various information that is likely to influence policy making: for instance, differences in the size and stage of development of the national economy could be accounted for by using different kinds of prior distributions $f(v)$ for the projects' values such that higher mean values and longer upper tails of $f(v)$ would be associated with larger and more developed economies.

The model could also be extended to cases in which $k \geq 2$ instead of just one interim evaluation may be acquired. The decision variables in such a multistage model would correspond to the number of projects that are, in the beginning of each stage, funded for their remaining duration, funded until the next evaluation stage, or abandoned. Then, the analytic results of Proposition 1 would hold for decisions made at any given stage of this process, but the exponentially increasing number of feasible combinations of decision variables would make it very time-consuming to compute optimal funding policies for larger values of $k$. To keep the computational effort at a reasonable level, optimality conditions such as that in Proposition 2 would need to be derived for multistage funding policies as well. 
Funding practices in which the continuation of funding is based on interim evaluation of projects are widely used by private companies (Sahlman, 1990; 770 Tellis et al., 2009; Klingebiel and Rammer, 2011). For example, when developing a new oil field, a firm may scale back planned investments to limit its downside exposure if oil prices, production rates, or reserves turn out to be below expectations (Smith and McCardle, 1999). Staged funding is also standard practice for venture capitalist firms (Tian, 2011; Li and Chi, 2013). This is

775 partly due to the need to establish incentives for the start-up entrepreneur to meet the stage targets (Gompers, 1995), but also due to the inability to predict accurately based on the business plan whether the start-up is going to be successful (Blank, 2013).

In comparison, many well-known research funding agencies which empha780 size the objective of promoting breakthrough research are effectively employing funding policies which, in our model, resemble those that maximize the expected portfolio value. Specifically, these agencies typically commit to funding all approved projects for a long time, e.g., for up to five years (Heinze, 2008), which also means that the process of launching projects is highly selective. Indeed, 785 the success rate of applications to the high-risk, high-reward programme of the NIH is 5\% (Gewin, 2012). Much in the same vein, the success rate for ERC starting grants has remained below $16 \%$ over the years, reaching an all-time low of $3.4 \%$ in $2007^{2}$.

Our model is generic and, hence, relevant to different project portfolio selection contexts. Yet, our model does not account for the specific details that appear in different project selection settings. For instance, we do not explicitly distinguish between different phases of technology development and commercialization, or between different public policy instruments for supporting breakthrough innovations. Furthermore, there may be important differences between research grants that are awarded based on the applicant's track record and those that are selected based on a peer review that is focused on the submitted applica-

${ }^{2}$ Source: http://erc.europa.eu/statistics-0, retrieved February 19, 2015. 
tion alone. There may also be administrative reasons for why private companies have embraced the abandonment option in their funding policies, while most research funding agencies have not. This notwithstanding, our model serves to highlight that breakthrough technologies can be best fostered by (i) experimenting by initiating a large set of technology projects, and (ii) committing resources only to those projects that, based on the experimentation, seem to have the potential to result in breakthroughs.

\section{Acknowledgments}

This research has been supported by the Finnish Funding Agency for Technology and Innovation (Tekes, 40419/11). We are grateful to editor Tugrul Daim and three anonymous reviewers for their insightful comments on the manuscript. We would also like to express our gratitude to the Academy of Finland (267503), the Finnish Science Foundation for Economics and Technology (KAUTE, 201300087) and the Marcus Wallenberg foundation (5-1507-12) for their financial support to Juuso Liesiö and Eeva Vilkkumaa.

\section{Appendix}

Proof of Proposition 1: Given the initial estimates $s_{i}^{0}$, the posterior distributions for the projects' future values $\left(V_{i} \mid S_{i}^{0}=s_{i}^{0}\right)$ are $f\left(v \mid s_{i}^{0}\right)=\mathrm{N}\left(v_{i}^{0}, \rho_{0}^{2}\right) \forall i \in \mathcal{N}$, where the variance $\rho_{0}^{2}=\sigma^{2} \tau_{0}^{2} /\left(\sigma^{2}+\tau_{0}^{2}\right)$ is the same for all projects. Prior to observing the interim estimates $s_{i}^{q}$, the projects' posterior distributions are $f\left(v \mid S_{i}^{q}, s_{i}^{0}\right)=\mathrm{N}\left(\mathbb{E}\left[V_{i} \mid S_{i}^{0}=\right.\right.$ $\left.\left.s_{i}^{0}, S_{i}^{q}\right], \rho_{q}^{2}\right)$, where the variance $\rho_{q}^{2}=r^{2 q} \tau_{0}^{2} \rho_{0}^{2} /\left(\rho_{0}^{2}+r^{2 q} \tau_{0}^{2}\right)$ is the same for all projects. The expected values are random variables $\mathbb{E}\left[V_{i} \mid S_{i}^{0}=s_{i}^{0}, S_{i}^{q}\right] \sim \mathrm{N}\left(v_{i}^{0}, \xi_{q}^{2}\right)$, where the variance $\xi_{q}^{2}=\rho_{0}^{4} /\left(\rho_{0}^{2}+r^{2 q} \tau_{0}^{2}\right)=\rho_{0}^{2}-\rho_{q}^{2}$ is the same for all projects.

We introduce random variables $X_{i}=\mathbb{E}\left[g\left(V_{i}\right) \mid S_{i}^{0}=s_{i}^{0}, S_{i}^{q}\right], i \in \mathcal{N}$, where $g$ : $\mathbb{R} \rightarrow \mathbb{R}$ is a non-decreasing function. Particularly, if $g(t)=g_{L}(t)=t$, then $X_{i}=$ $\mathbb{E}\left[V_{i} \mid S_{i}^{0}=s_{i}^{0}, S_{i}^{q}\right]$, and by the law of total expectation $\mathbb{E}_{S_{i}^{q}}\left[X_{i}\right]=\mathbb{E}_{S_{i}^{q}}\left[\mathbb{E}\left[V_{i} \mid S_{i}^{0}=\right.\right.$ $\left.\left.s_{i}^{0}, S_{i}^{q}\right]\right]=\mathbb{E}\left[V_{i} \mid S_{i}^{0}=s_{i}^{0}\right]$. If, in turn, $g(t)$ is the step function

$$
g_{S}(t)=\left\{\begin{array}{cc}
0 & \text { for all } t<\mu_{\alpha}^{*} \\
1 & \text { for all } t \geq \mu_{\alpha}^{*},
\end{array}\right.
$$


then $X_{i}=\mathbb{P}\left(V_{i} \geq \mu_{\alpha}^{*} \mid S_{i}^{0}=s_{i}^{0}, S_{i}^{q}\right)$, and by the law of total expectation $\mathbb{E}_{S_{i}^{q}}\left[X_{i}\right]=$ $\mathbb{E}\left[g_{S}\left(V_{i}\right) \mid S_{i}^{0}=s_{i}^{0}\right]=\mathbb{P}\left(V_{i} \geq \mu_{\alpha}^{*} \mid S_{i}^{0}=s_{i}^{0}\right)$.

Using this notation, we can define a general portfolio selection problem for a fixed funding policy $(\ell, e, a, q)$ and a set of fixed initial estimates $s_{i}^{0}, i \in \mathcal{N}$ :

$$
\left.\max _{\substack{\mathcal{L} \subseteq \mathcal{N}, \mathcal{E} \subseteq \mathcal{L} \\|\mathcal{L}|=\ell, \mathcal{E} \mid=e}}\left\{\sum_{i \in \mathcal{L} \backslash \mathcal{E}} \mathbb{E}_{S_{i}^{q}}\left[X_{i}\right]+\mathbb{E}_{S^{q}}\left[\max _{\substack{\mathcal{A} \subseteq \mathcal{E} \\|\mathcal{A}|=a}}\left\{\sum_{i \in \mathcal{E} \backslash \mathcal{A}} X_{i}+h(q) \sum_{i \in \mathcal{A}} X_{i}\right]\right\}\right]\right\}
$$

which corresponds to the inner problem of $(7)$ if $g=g_{L}$ and $h:\{1, \ldots, d-1\} \rightarrow[0,1)$, and to the inner problem of (8) if $g=g_{S}$ and $h(q) \equiv 0$. To prove the proposition, it is thus sufficient to show that (9)-(11) hold for Problem (12) for any feasible policy $(\ell, e, a, q)$. To accomplish this we establish that

$$
v_{k}^{0}>v_{j}^{0} \Leftrightarrow \mathbb{E}_{S_{k}^{q}}\left[X_{k}\right]>\mathbb{E}_{S_{j}^{q}}\left[X_{j}\right] \Leftrightarrow F_{X_{k}}(x) \subsetneq F_{X_{j}}(x) \forall x
$$

which follows from the fact that the cumulative distribution function of $X_{i}$ is $F_{X_{i}}(x)=$ $\Phi\left(\left(x-v_{i}^{0}\right) / \xi_{q}\right)$ if $g=g_{L}$, and

$$
F_{X_{i}}(x)= \begin{cases}0, & x \leq 0, \\ \Phi\left(\frac{\mu_{\alpha}^{*}+\rho_{q} \Phi^{-1}(x)-v_{i}^{0}}{\xi_{q}}\right), & 0<x<1, \\ 1, & x \geq 1,\end{cases}
$$

if $g=g_{S}$ (Taboga, 2012).

Proof of (9) Take any $\hat{\mathcal{L}} \subseteq \mathcal{N}$ and $\hat{\mathcal{E}} \subseteq \hat{\mathcal{L}}$, such that $|\hat{\mathcal{L}}|=\ell$ and $|\hat{\mathcal{E}}|=e$. When choosing which project to abandon (i.e., $\mathcal{A}$ ), the interim estimates $s_{i}^{q}$ are known so that also the realizations of the random variables $X_{i}=\mathbb{E}\left[g\left(V_{i}\right) \mid S_{i}^{0}=s_{i}^{0}, S_{i}^{q}\right], i \in \hat{\mathcal{E}}$ are known; denote these by $x_{i}=\mathbb{E}\left[g\left(V_{i}\right) \mid S_{i}^{0}=s_{i}^{0}, S_{i}^{q}=s_{i}^{q}\right], i \in \hat{\mathcal{E}}$. Take any $\hat{\mathcal{A}} \subseteq \hat{\mathcal{E}}$ such that $|\hat{\mathcal{A}}|=a$, and assume there exist $j \in \hat{\mathcal{E}} \backslash \hat{\mathcal{A}}$ and $k \in \hat{\mathcal{A}}$ such that $x_{k}>x_{j}$. Then, evaluating the objective function of the inner maximization of problem in (12) at (i) $\mathcal{A}=\hat{\mathcal{A}}$ and at (ii) $\mathcal{A}=\hat{\mathcal{A}} \backslash\{k\} \cup\{j\}$, and computing the difference between these 830 values gives

$$
\begin{aligned}
& \overbrace{\sum_{i \in \hat{\mathcal{E}} \backslash \hat{\mathcal{A}}} x_{i}+h(q) \sum_{i \in \hat{\mathcal{A}}} x_{i}}^{(\mathrm{i})}-\overbrace{\left.\sum_{i \in(\hat{\mathcal{E}} \backslash \hat{\mathcal{A}}) \backslash\{j\} \cup\{k\}} x_{i}+h(q) \sum_{i \in \hat{\mathcal{A}} \backslash\{k\} \cup\{j\}} x_{i}\right]}^{\text {(ii) }}= \\
& x_{j}+h(q) x_{k}-x_{k}-h(q) x_{j}=(1-h(q))\left(x_{j}-x_{k}\right)<0,
\end{aligned}
$$

where the inequality is implied by the assumption that $x_{k}>x_{j}$ and the fact that $h(q) \in[0,1)$. Hence, the objective function could be improved by abandoning project $j$ instead of $k$. 
Proof of (10). Take any $\hat{\mathcal{L}} \subseteq \mathcal{N}$ and $\hat{\mathcal{E}} \subseteq \hat{\mathcal{L}}$ such that $|\hat{\mathcal{L}}|=\ell$ and $|\hat{\mathcal{E}}|=e$. Assume there exist $j \in \hat{\mathcal{L}} \backslash \hat{\mathcal{E}}$ and $k \in \hat{\mathcal{E}}$ such that $v_{k}^{0}>v_{j}^{0}$. Let $X_{(1)} \geq \ldots \geq X_{(e-1)}$ be the order statistics of the random variables $X_{i}, i \in \hat{\mathcal{E}} \backslash\{k\}$ and let $P_{\underline{s}: \bar{s}}=\sum_{i=\underline{s}}^{\bar{s}} X_{(i)}$. Then, evaluating the objective function of problem (12) at (i) $\mathcal{L}=\hat{\mathcal{L}}, \mathcal{E}=\hat{\mathcal{E}}$ and at (ii) $\mathcal{L}=\hat{\mathcal{L}}, \mathcal{E}=\hat{\mathcal{E}} \backslash\{k\} \cup\{j\}$, and computing the difference gives

(i)

$$
\overbrace{\sum_{i \in \hat{\mathcal{L}} \backslash \hat{\mathcal{E}}} \mathbb{E}_{S_{i}^{q}}\left[X_{i}\right]+\mathbb{E}_{S^{q}}\left[\max \left\{P_{1: e-a}+h(q)\left(X_{k}+P_{e-a+1: e-1}\right),\left(P_{1: e-a-1}+X_{k}\right)+h(q) P_{e-a: e-1}\right\}\right]}
$$

(ii)

$$
\begin{aligned}
& -\overbrace{\sum_{i \in(\hat{\mathcal{L}} \backslash \hat{\mathcal{E}}) \backslash\{j\} \cup\{k\}} \mathbb{E}_{S_{i}^{q}}\left[X_{i}\right]-\mathbb{E}_{S^{q}}\left[\max \left\{P_{1: e-a}+h(q)\left(X_{j}+P_{e-a+1: e-1}\right),\left(P_{1: e-a-1}+X_{j}\right)+h(q) P_{e-a: e-1}\right\}\right]}^{=}= \\
& \mathbb{E}_{S^{q}}\left[X_{j}+P_{1: e-a-1}+h(q) P_{e-a+1: e-1}+\max \left\{X_{(e-a)}+h(q) X_{k}, h(q) X_{(e-a)}+X_{k}\right\}\right] \\
& -\mathbb{E}_{S^{q}}\left[X_{k}+P_{1: e-a-1}+h(q) P_{e-a+1: e-1}+\max \left\{X_{(e-a)}+h(q) X_{j}, h(q) X_{(e-a)}+X_{j}\right\}\right]= \\
& \mathbb{E}_{S^{q}}\left[\max \left\{X_{(e-a)}+h(q) X_{k}, h(q) X_{(e-a)}+X_{k}\right\}-X_{k}\right]-\mathbb{E}_{S q}\left[\max \left\{X_{(e-a)}+h(q) X_{j}, h(q) X_{(e-a)}+X_{j}\right\}-X_{j}\right]= \\
& \mathbb{E}_{S^{q}}\left[\max \left\{X_{(e-a)}-(1-h(q)) X_{k}, h(q) X_{(e-a)}\right\}\right]-\mathbb{E}_{S^{q}}\left[\max \left\{X_{(e-a)}-(1-h(q)) X_{j}, h(q) X_{(e-a)}\right\}\right]= \\
& \mathbb{E}_{S^{q}}\left[\max \left\{(h(q)-1) X_{k},(h(q)-1) X_{(e-a)}\right\}+X_{(e-a)}\right]-\mathbb{E}_{S^{q}}\left[\max \left\{(h(q)-1) X_{j},(h(q)-1) X_{(e-a)}\right\}+X_{(e-a)}\right]= \\
& \underbrace{(h(q)-1)}_{<0}(\underbrace{\mathbb{E}_{S q}\left[\min \left\{X_{k}, X_{(e-a)}\right\}\right]-\mathbb{E}_{S^{q}}\left[\min \left\{X_{j}, X_{(e-a)}\right\}\right]}_{>0 \text { by }(13)})<0 .
\end{aligned}
$$

Hence the objective function value could be improved by evaluating project $j$ instead

840 of $k$.

Proof of (11). Take any $\hat{\mathcal{L}} \subseteq \mathcal{N}$ and $\hat{\mathcal{E}} \subseteq \hat{\mathcal{L}}$, such that $|\hat{\mathcal{L}}|=\ell$ and $|\hat{\mathcal{E}}|=e$. Assume there exists $j \in \hat{\mathcal{L}}$ and $k \notin \hat{\mathcal{L}}$ such that $v_{k}^{0}>v_{j}^{0}$.

First, assume $j \notin \hat{\mathcal{E}}$. Let $X_{(1)} \geq \ldots \geq X_{(|\mathcal{E}|)}$ be the order statistics of the random variables $X_{i}, i \in \hat{\mathcal{E}}$ and let $P_{\underline{s}: s}=\sum_{i=\underline{s}}^{\bar{s}} X_{(i)}$. Evaluating the objective function of problem (12) at (i) $\mathcal{L}=\hat{\mathcal{L}}, \mathcal{E}=\hat{\mathcal{E}}$ and at (ii) $\mathcal{L}=\hat{\mathcal{L}} \backslash\{j\} \cup\{k\}, \mathcal{E}=\hat{\mathcal{E}}$, and computing the difference gives

$$
\overbrace{\sum_{i \in \hat{\mathcal{L}} \backslash \hat{\mathcal{E}}} \mathbb{E}_{S_{i}^{q}}\left[X_{i}\right]+\mathbb{E}_{S^{q}}\left[P_{1: e-a}+h(q) P_{e-a+1: e}\right]}^{\text {(i) }}-\overbrace{\left[\sum_{i \in(\hat{\mathcal{L}} \backslash \hat{\mathcal{E}}) \backslash\{j\} \cup\{k\}} \mathbb{E}_{S_{i}^{q}}\left[X_{i}\right]+\mathbb{E}_{S^{q}}\left[P_{1: e-a}+h(q) P_{e-a+1: e}\right]\right]}^{(\mathrm{ii})}=\mathbb{E}_{S_{j}^{q}\left[X_{j}\right]-\mathbb{E}_{S_{k}^{q}}\left[X_{k}\right]}<0,
$$

where the inequality is implied by (13). Hence the objective function value could be improved by launching project $k$ instead of $j$.

Second, assume that $j \in \hat{\mathcal{E}}$. Let $X_{(1)} \geq \ldots \geq X_{(e-1)}$ be the order statistics of the random variables $X_{i}, i \in \hat{\mathcal{E}} \backslash\{j\}$ and let $P_{\underline{s}: \bar{s}}=\sum_{i=\underline{s}}^{\bar{s}} X_{(i)}$. Then, evaluating the objective function of problem (12) at (i) $\mathcal{L}=\hat{\mathcal{L}}, \mathcal{E}=\hat{\mathcal{E}}$ and at (ii) $\mathcal{L}=\hat{\mathcal{L}} \backslash\{j\} \cup\{k\}$, 
$\mathcal{E}=\hat{\mathcal{E}} \backslash\{j\} \cup\{k\}$, and computing the difference gives

$$
\overbrace{\sum_{i \in \hat{\mathcal{L}} \backslash \hat{\mathcal{E}}} \mathbb{E}_{S_{i}^{q}}\left[X_{i}\right]+\mathbb{E}_{S^{q}}\left[\max \left\{P_{1: e-a-1}+X_{j}+h(q) P_{e-a: e-1}, P_{1: e-a}+h(q)\left(P_{e-a+1: e-1}+X_{j}\right)\right\}\right]}^{(\mathrm{i})}-
$$

(ii)

$$
\begin{aligned}
-\overbrace{\sum_{i \in \hat{\mathcal{L}} \backslash \hat{\mathcal{E}}} \mathbb{E}_{S_{i}^{q}}\left[X_{i}\right]-\mathbb{E}_{S^{q}}\left[\max \left\{P_{1: e-a-1}+X_{k}+h(q) P_{e-a: e-1}, P_{1: e-a}+h(q)\left(P_{e-a+1: e-1}+X_{k}\right)\right\}\right]} & = \\
\mathbb{E}_{S^{q}}\left[\max \left\{X_{j}+h(q) X_{(e-a)}, X_{(e-a)}+h(q) X_{j}\right\}-\max \left\{X_{k}+h(q) X_{(e-a)}, X_{(e-a)}+h(q) X_{k}\right\}\right] & = \\
h(q) \underbrace{\mathbb{E}_{S^{q}}\left[X_{j}-X_{k}\right]}_{<0 \text { by }(13)}+(1-h(q)) \underbrace{\mathbb{E}_{S^{q}}\left[\max \left\{X_{j}, X_{(e-a)}\right\}-\max \left\{X_{k}, X_{(e-a)}\right\}\right]}_{<0 \text { by }(13)} & <0
\end{aligned}
$$

where the inequality is implied by fact that $h(q) \in[0,1)$. Hence the objective function value could be improved by launching and evaluating project $k$ instead of $j \square$.

Proof of Proposition 2: Let us use the same notation as in the proof of Proposition 1. Let $(\ell, e, a, q)$ be the optimal solution to (12), which represents both problem (7) with $g=g_{L}, h:\{1, \ldots, d-1\} \rightarrow[0,1)$ and problem (8) with $g=g_{S}, h(q) \equiv 0$. To prove the proposition, it is thus sufficient to show that policy $(\ell, e+1, a, q)$ is infeasible for (12). Assume to the contrary that $(\ell, e+1, a, q)$ is feasible for (12). Let $\hat{\mathcal{L}}$ and $\hat{\mathcal{E}}$ be the optimal index sets for the optimal policy $(\ell, e, a, q)$ such that $j \in \hat{\mathcal{L}} j \notin \hat{\mathcal{E}}$. Let $X_{(1)} \geq \ldots \geq X_{(e)}$ be the order statistics of the random variables $X_{i}, i \in \hat{\mathcal{E}}$ and let $P_{\underline{s}: \bar{s}}=\sum_{i=s}^{\bar{s}} X_{(i)}$. Then, evaluating the objective function of problem (12) at (i) $\mathcal{L}=\hat{\mathcal{L}}, \mathcal{E}=\hat{\mathcal{E}}$ and at (ii) $\mathcal{L}=\hat{\mathcal{L}}, \mathcal{E}=\hat{\mathcal{E}} \cup\{j\}$ (i.e., $|\mathcal{E}|=e+1$ ), and computing the difference gives

(ii)

$$
\begin{aligned}
& \overbrace{\sum_{i \in \hat{\mathcal{L}} \backslash(\hat{\mathcal{E}} \cup\{j\})} \mathbb{E}_{S_{i}^{q}}\left[X_{i}\right]+\mathbb{E}_{S^{q}}\left[\max \left\{P_{1: e-a}+X_{j}+h(q) P_{e-a+1: e}, P_{1: e-a+1}+h(q)\left(P_{e-a+2: e}+X_{j}\right)\right\}\right]}^{-}- \\
& -\overbrace{\sum_{i \in \hat{\mathcal{L}} \backslash \hat{\mathcal{E}}} \mathbb{E}_{S_{i}^{q}}\left[X_{i}\right]-\mathbb{E}_{S^{q}}\left[P_{1: e-a}+h(q) P_{e-a+1: e}\right]}^{(\mathrm{i})}= \\
& \mathbb{E}_{S_{i}^{q}}\left[\operatorname { m a x } \left\{P_{1: e-a}+X_{j}+h(q) P_{e-a+1: e}-X_{j}-P_{1: e-a}-h(q) P_{e-a+1: e},\right.\right. \\
& \left.\left.P_{1: e-a+1}+h(q)\left(P_{e-a+2: e}+X_{j}\right)-X_{j}-P_{1: e-a}-h(q) P_{e-a+1: e}\right\}\right]= \\
& \underbrace{(1-h(q))}_{>0} \mathbb{E}_{S^{q}}\left[\max \left\{0, X_{(e-a+1)}-X_{j}\right\}\right]>0,
\end{aligned}
$$

where the last inequality follows from the fact that $X_{(e-a+1)}>X_{j}$ with a positive 
probability. This, however, contradicts the assumption that policy $(\ell, e, a, q)$ is optimal for (12), whereby $(\ell, e+1, a, q)$ must be infeasible $\square$.

\section{References}

Adner, R., Levinthal, A., 2002. The emergence of emerging technologies. Technical Report. Insead.

Alvarez, L., 2001. Reward functionals, salvage values and optimal stopping. Mathematical Methods of Operations Research 154, 315-337.

Arthur, B., 2009. The Nature of Technology: What It Is and How It Evolves. Penguin Books, London.

875 Azoulay, P., Zivin, J.G., Manso, G., 2011. Incentives and creativity: evidence from the academic life sciences. The RAND Journal of Economics 42, 527-554.

Blank, S., 2013. Why the lean start-up changes everything. Harvard Business Review $91,64-73$.

Boehm, B.W., 1981. Software Engineering Economics. Prentice Hall, New Jersey.

Borrás, S., Equist, C., 2013. The choice of innovation policy instruments. Technological Forecasting \& Social Change 80, 1513-1522.

Bourke, P., Butler, L., 1999. The efficacy of different modes of funding research: perspectives from Australian data on the biological sciences. Research Policy 28, 489-499.

Brown, R., 1992. Managing the "S" curves of technology. Journal of Business \& Industrial Marketing 7, 41-52.

Christensen, C.M., 1997. The Innovator's Dilemma: When New Technologies Cause Great Firms to Fail. Harvard Business School Press, Cambridge.

Dixit, A., Pindyck, R., 1994. Investment Under Uncertainty. Princeton Press, Princeton.

ERC, 2014. Misson statement of the ERC. Retrieved 22 Oct 2014, http://erc.europa.eu/about-erc/mission. ERC. 
Gewin, V., 2012. Risky research: The sky's the limit. Nature 487, 395-397.

Godin, B., 2005. The linear model of innovation the historical construction of an analytical framework. Science, Technology \& Human Values 31, 639-667.

Gompers, P., 1995. Optimal investment, monitoring, and the staging of venture capital. Journal of Finance 50, 1461-1489.

Gustafsson, J., Salo, A., 2005. Contingent portfolio programming for the management of risky projects. Operations Research 53, 946-956.

900 Hall, B., Lerner, J., 2009. The Financing of R\&D and Innovation. National Bureau of Economic Research, Cambridge.

Heinze, T., 2008. How to support ground-breaking research: a comparison of funding schemes. Science and Public Policy 35, 302-318.

Huchzermeier, A., Loch, C.H., 2001. Project management under risk: using the real options approach to evaluate flexibility in R\&D. Management Science 47, 85-101.

Klingebiel, R., Rammer, C., 2011. Resource allocation flexibility for innovation performance: the effects of breadth, uncertainty, and selectiveness. Centre for European Economic Research, Discussion paper No. 11-073.

Kwon, H.D., Lippman, S.A., 2011. Acquisition of project-specific assets with Bayesian updating. Operations Research 59, 1119-1130.

Lerner, J., 2009. Boulevard of Broken Dreams. Princeton University Press, Princeton.

Li, Y., Chi, T., 2013. Venture capitalists' decision to withdraw: The role of portfolio configuration from a real options lens. Strategic Management Journal 35, 1351-1366.

Liesiö, J., Mild, P., Salo, A., 2008. Robust portfolio modeling with incomplete cost information and project interdependencies. European Journal of Operational Research 190, 679-695.

Marxt, C., Brunner, C., 2013. Analyzing and improving the national innovation system of highly developed countries - the case of Switzerland. Technological Forecasting \& Social Change 80, 1035-1049. 
Melin, G., Danell, R., 2006. The top eight percent: development of approved and rejected applicants for a prestigious grant in Sweden. Science and Public Policy 33, $702-712$.

NIH, 2014. National Institues of Health high-risk research program overview. Retrieved 22 Oct 2014, http://commonfund.nih.gov/highrisk/overview.aspx. NIH.

925 O'Connor, G.C., Ravichandran, T., Robeson, D., 2008. Risk management through learning: Management practices for radical innovation success. The Journal of High Technology Management Research 19, 70-82.

OECD, 2010. The Impacts of Nanotehcnology on Companies: Policy Insights from Case Studies. OECD, Paris.

Öquist, G., Benner, M., 2012. Fostering Breakthrough Research: A Comparative Study. The Royal Swedish Academy of Sciences, Stockholm.

Pasternack, B.A., 1985. Optimal pricing and return policies for perishable commodities. Management Science 4, 166-176.

Porter, A., Ashton, W., Clar, G., Coates, J., Cuhls, K., Cunningham, A., Ducatel, K., van der Duin, P., Georgehiou, L., Gordon, T., Linstone, H., Marcahu, V., Massari, G., Miles, I., Mogee, M., Salo, A., Scapolo, F., Smits, R., Thissen, W., 2004. Technology futures analysis: toward integration of the field and new methods. Technological Forecasting \& Social Change 71, 287-303.

Roberts, K., Weitzman, M., 1981. Funding criteria for research, development, and exploration projects. Econometrica 49, 1261-1288.

Sahlman, W.A., 1990. The structure and governance of venture-capital organizations. Journal of Financial Economics 27, 473-521.

Salo, A., Keisler, J., Morton, A., 2011. An invitation to portfolio decision analysis, in: Salo, A., Keisler, J., Morton, A. (Eds.), Portfolio Decision Analysis: Improved Methods for Resource Allocation. Springer, New York.

Santiago, L.P., Vakili, P., 2005. On the value of flexibility in R\&D projects. Management Science 51, 1206-1218. 
Schilling, M.A., 2008. Strategic Management of Technological Innovation. McGrawHill, Singapore.

Sharpe, S., Cosh, A., Connell, D., 2013. Funding Breakthrough Technology. Cambridge Integrated Knowledge Centre report, Cambridge.

Siddiqui, A.S., Fleten, S.E., 2010. How to proceed with competing alternative energy technologies: A real options approach. Energy Economics 32, 817-830.

Siddiqui, A.S., Marnay, C., Wiser, R.H., 2007. Real options valuation of US federal renewable energy research, development, demonstration, and deployment. Energy Policy 35, 265-279.

Smith, J.E., McCardle, K.F., 1999. Options in the real world: lessons learned in evaluating oil and gas investments. Operations Research 47, 1-15.

Taboga, M., 2012. Lectures on Probability Theory and Mathematical Statistics - 2nd Edition. CreateSpace Independent Publishing Platform.

Teece, D.E., 1986. Profiting from technological innovation: Implications for integration, collaboration, licensing and public policy. Research Policy 15, 285-305.

Tellis, G.J., Prabhu, J.C., Chandy, R.K., 2009. Radical innovation across nations: the preeminence of corporate culture. Journal of Marketing 15, 3-23.

Tian, X., 2011. The causes and consequences of venture capital stage financing. Journal of Financial Economics 101, 132-159.

Utterback, J., 1994. Mastering the Dynamics of Innovation: How Companies Can Seize Opportunities in the Face of Technological Change. Harvard Business School Press, Boston.

Vilkkumaa, E., Liesiö, J., Salo, A., 2014. Optimal strategies for selecting project portfolios using uncertain value estimates. European Journal of Operational Research $233,772-783$.

Walk, S., 2012. Quantitative technology forecasting techniques, in: Teixeira, A. (Ed.), Technological Change. InTech, http://www.intechopen.com/books/technologicalchange. 


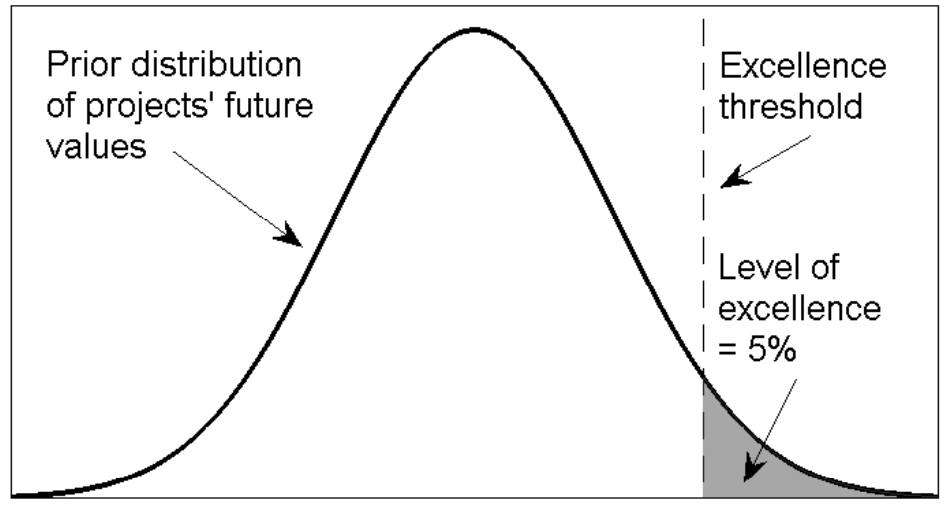

Figure 1: Illustration of the excellence threshold. 


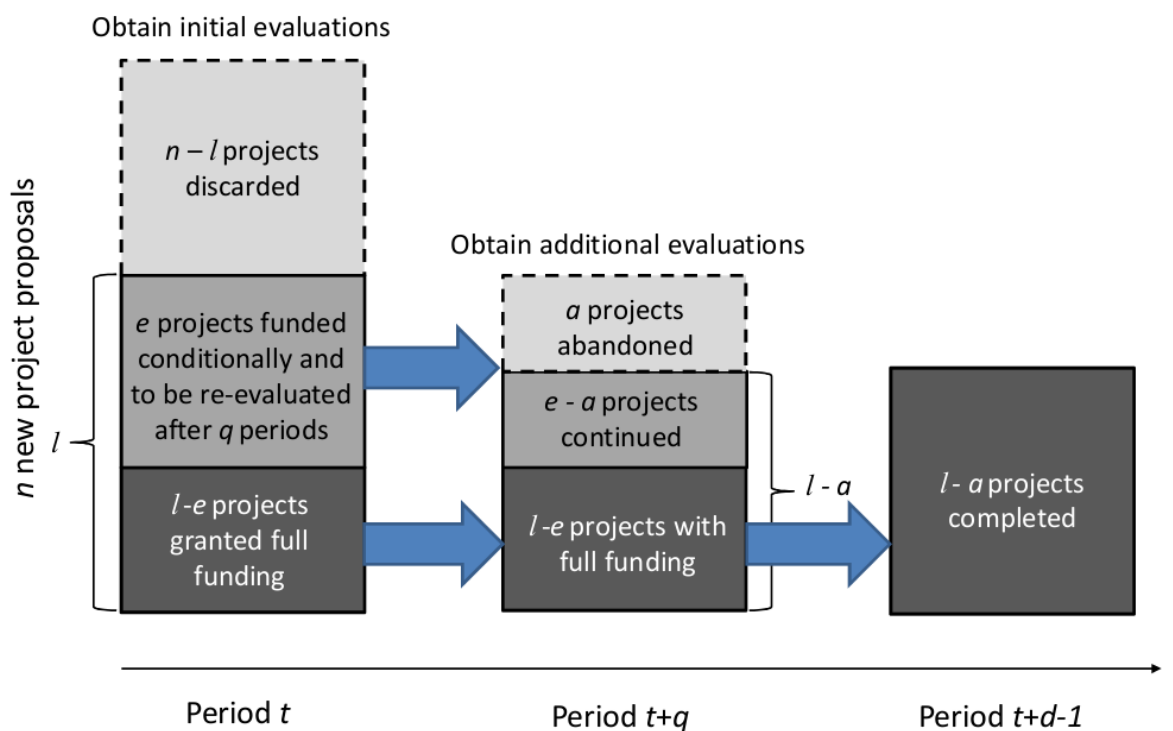

Figure 2: Portfolio selection process for project proposals arriving at the beginning of period $t$. 


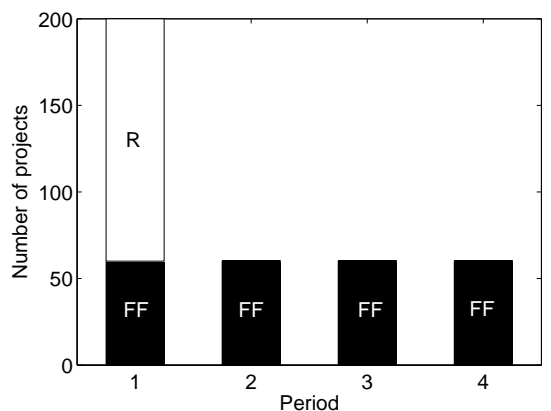

(a) Accurate estimates, expected value

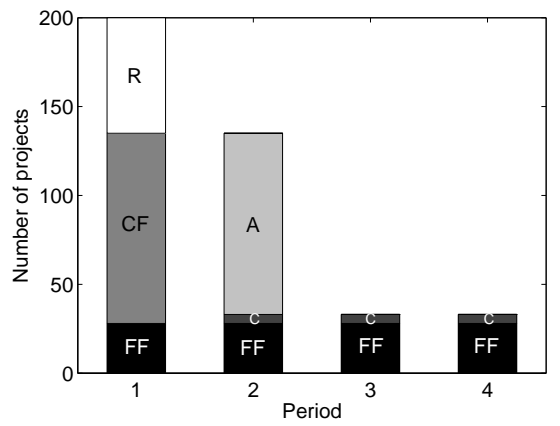

(c) Accurate estimates, exceptional projects

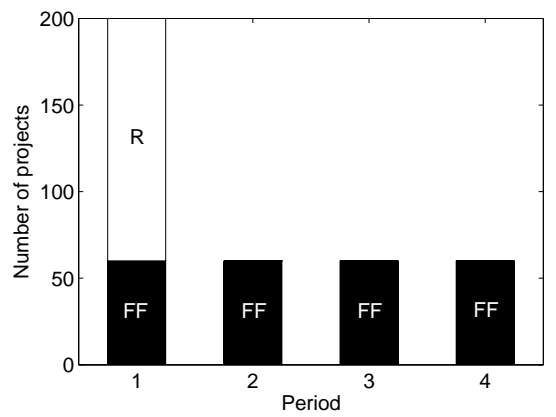

(b) Inaccurate estimates, expected value

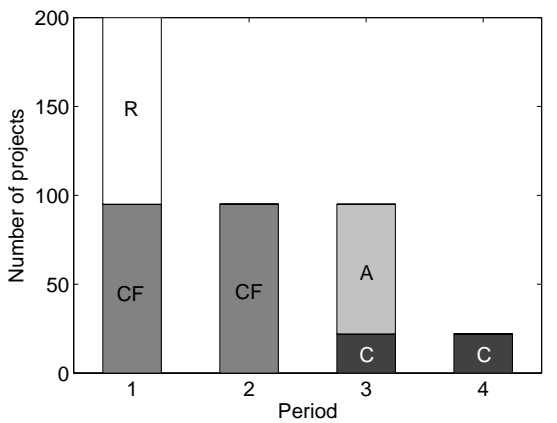

(d) Inaccurate estimates, exceptional projects

Figure 3: The optimal funding policies when the objective is to maximize the expected portfolio value (a)-(b), or the expected number of funded projects among the top $1 \%$ (c)-(d). 5,000 simulation rounds (Monte Carlo). 


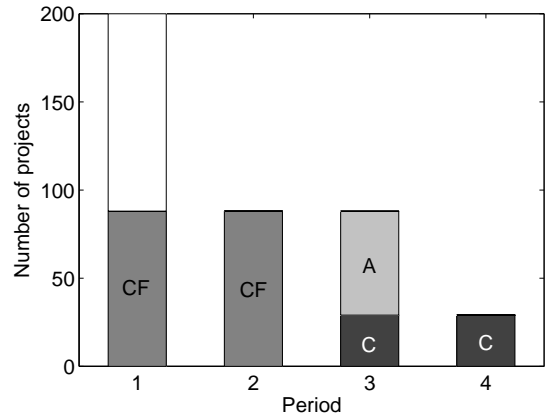

(a) Top $10 \%$, accuracy improves quickly

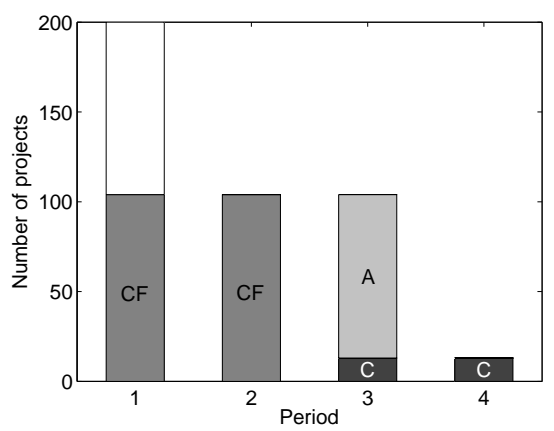

(c) Top $1 \%$, accuracy improves quickly

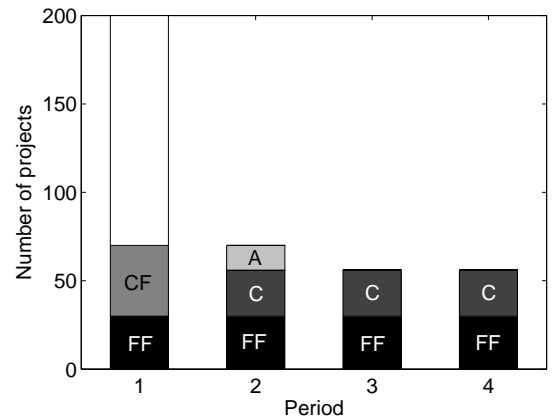

(b) Top 10\%, accuracy improves slowly

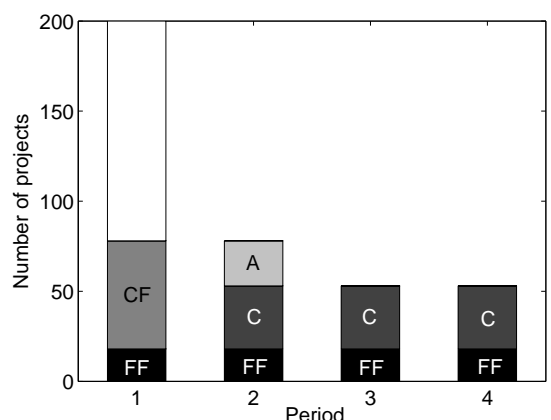

(d) Top 1\%, accuracy improves slowly

Figure 4: The optimal funding policies for fast $(r=0.4)$ or slow $(r=0.8)$ rate of uncertainty reduction, when the objective is to maximize the expected number of funded projects among the top $10 \%$ or top $1 \%$. 5,000 simulation rounds (Monte Carlo). 


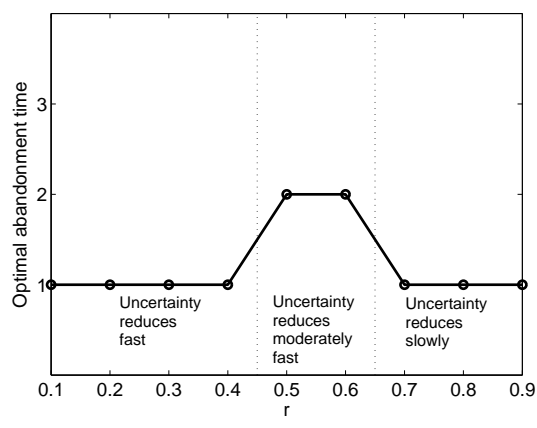

(a) Moderately inaccurate estimates $\tau_{0}=6$

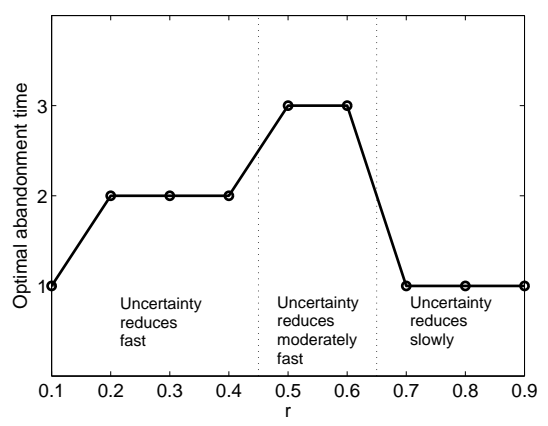

(b) Very inaccurate estimates $\tau_{0}=13$

Figure 5: Optimal abandonment time $q$ for maximizing the expected number of exceptional projects. 5,000 simulation rounds (Monte Carlo). 


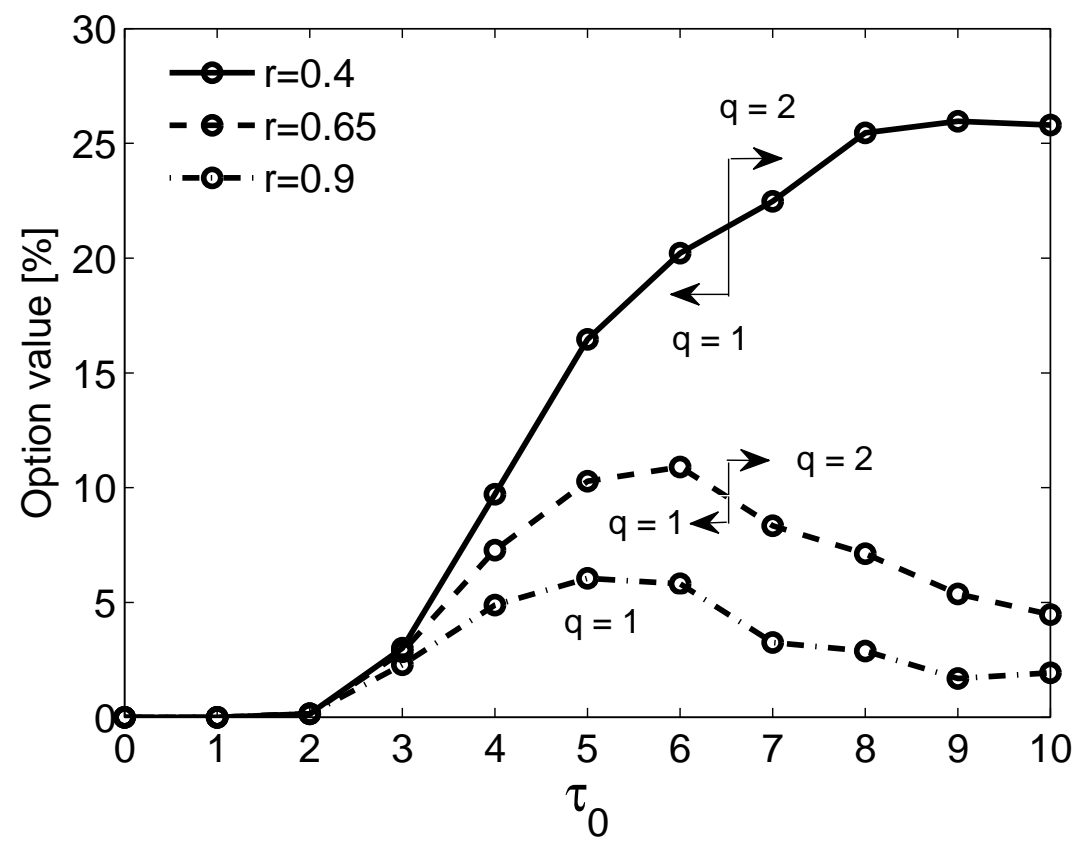

Figure 6: Value of the abandonment option in maximizing the expected number of exceptional projects for different values of $\tau_{0}$ and $r .5,000$ simulation rounds (Monte Carlo). 


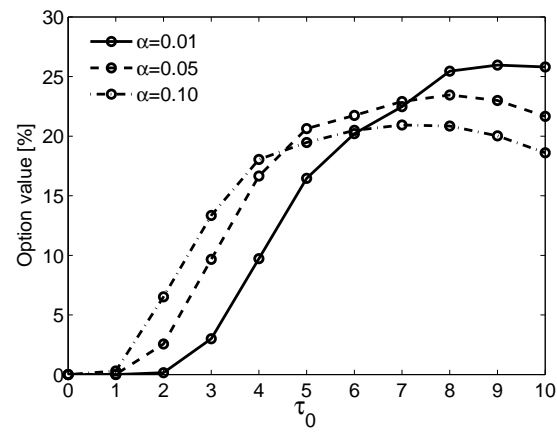

(a) Uncertainty reduces quickly $(r=0.4)$

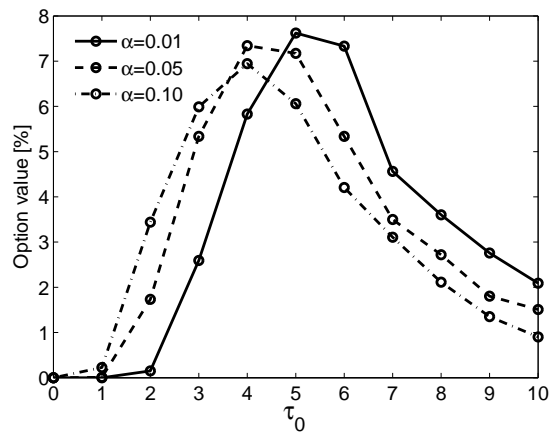

(b) Uncertainty reduces slowly $(r=0.8)$

Figure 7: Value of the abandonment option for different values of $\tau_{0}$ and $\alpha .5,000$ simulation rounds (Monte Carlo). 


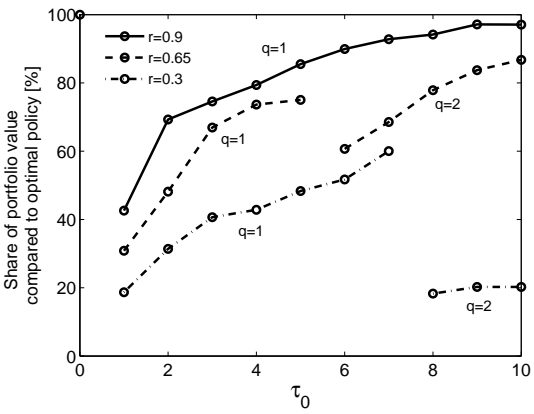

(a) Share of expected portfolio value

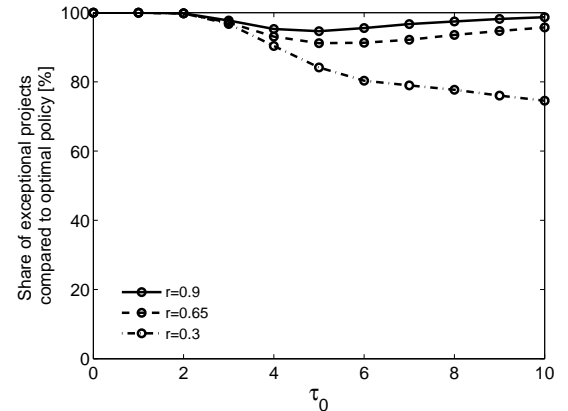

(b) Share of exceptional projects

Figure 8: (a): The percentage of the expected portfolio value resulting from a policy that maximizes the expected number of exceptional projects compared to a policy that maximizes this value. (b): The percentage of exceptional projects funded with a policy that maximizes the expected portfolio value compared to a policy that maximizes the expected number of exceptional projects. 5,000 simulation rounds (Monte Carlo). 


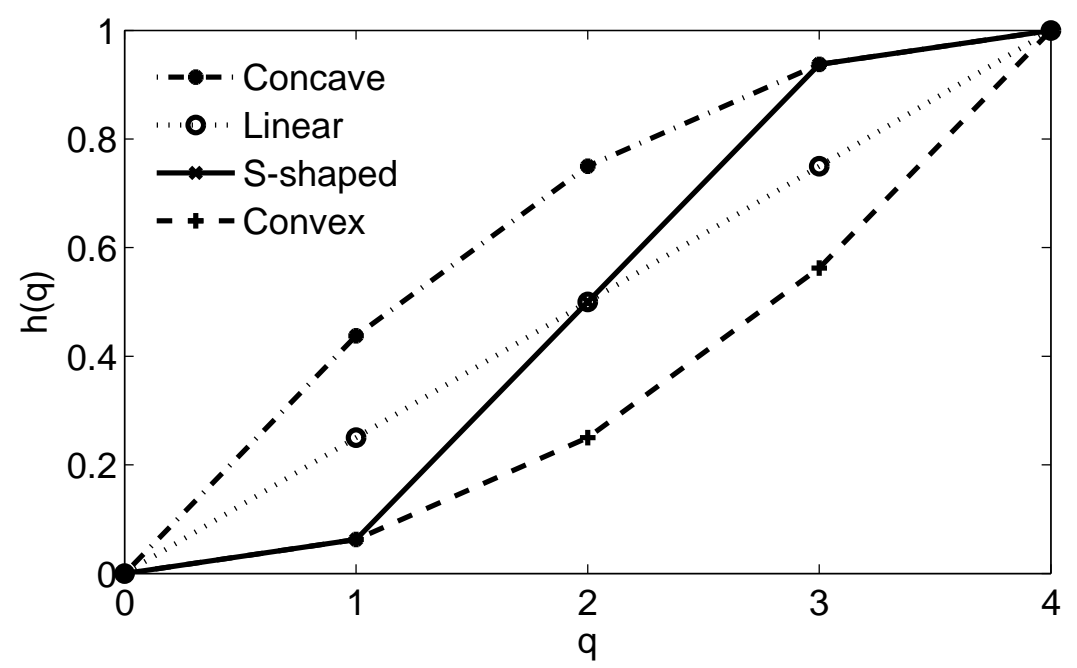

Figure 9: Different shapes of the salvage value function $h(q)$. 